\title{
Development of an Aerodynamic Analysis Method and Database for the SLS Service Module Panel Jettison Event Utilizing Inviscid CFD and MATLAB ${ }^{\circledR}$
}

\author{
Michael P. Applebaum ${ }^{1}$, Leslie H. Hall ${ }^{2}$ and William M. Eppard ${ }^{1}$ \\ CRM Solutions Inc., Jacobs ESSSA Group, Huntsville, AL, 35802 \\ David C. Purinton ${ }^{3}$ \\ Gray Research Inc., Jacobs ESSSA Group, Huntsville, AL, 35806 \\ John R. Campbell Jr. ${ }^{4}$ and John A. Blevins ${ }^{5}$ \\ NASA Marshall Space Flight Center, Huntsville, AL, 35812
}

\begin{abstract}
This paper describes the development, testing, and utilization of an aerodynamic force and moment database for the Space Launch System (SLS) Service Module (SM) panel jettison event. The database is a combination of inviscid Computational Fluid Dynamic (CFD) data and MATLAB code written to query the data at input values of vehicle/SM panel parameters and return the aerodynamic force and moment coefficients of the panels as they are jettisoned from the vehicle. The database encompasses over 5000 CFD simulations with the panels either in the initial stages of separation where they are hinged to the vehicle, in close proximity to the vehicle, or far enough from the vehicle that body interference effects are neglected. A series of viscous CFD check cases were performed to assess the accuracy of the Euler solutions for this class of problem and good agreement was obtained. The ultimate goal of the panel jettison database was to create a tool that could be coupled with any 6-Degree-Of-Freedom (DOF) dynamics model to rapidly predict SM panel separation from the SLS vehicle in a quasi-unsteady manner. Results are presented for panel jettison simulations that utilize the database at various SLS flight conditions. These results compare favorably to an approach that directly couples a 6-DOF model with the Cart3D Euler flow solver and obtains solutions for the panels at exact locations. This paper demonstrates a method of using inviscid CFD simulations coupled with a 6-DOF model that provides adequate fidelity to capture the physics of this complex multiple moving-body panel separation event.
\end{abstract}

\section{Introduction}

DeVElOPMENT of the Space Launch System (SLS) Service Module (SM) panel jettison database is part of an ongoing effort in support of the SLS SM panel separation and clearance analysis. The goal of this program is to determine appropriate jettison criteria, including separation timing, flight conditions, and mechanical separation forces, to ensure that safe panel jettison will occur without recontact. The approach and conclusions in this analysis build upon prior analysis for a similar class problem in which time accuracy versus quasi-steady state approach was examined $^{1,2}$. The critical component for accurate prediction of panel trajectory is proper capturing of the physics at the initial time of separation of the panel from the body. It has been shown that for bodies beginning at rest and undergoing moderate acceleration, quasi-unsteady techniques can provide adequate predictive capabilities ${ }^{3}$. As an

\footnotetext{
${ }^{1}$ Senior Research Scientist.

${ }^{2}$ President, Member AIAA.

${ }^{3}$ Principal Engineer.

${ }^{4}$ Aerospace Engineer, Aerosciences Branch.

${ }^{5}$ Division Technical Assistant, Aerosciences Branch, Senior Member AIAA.
} 
illustration, captive trajectory data from the wind tunnel testing has often been a basis for comparison and validation for fully time accurate moving-body CFD solvers ${ }^{4}$.

For this class of problem, initial studies at expected flight conditions demonstrated that neglecting the vehicle body when computing aerodynamic coefficients on the panel resulted in incorrect prediction of the panel trajectory. As the panels separate from the body, the cavity beneath the panels undergoes pressurization from the aerodynamic environment. The result is increased angular momentum being imparted to the panels which actually assists in the early stages of the separation event by increasing the distance the panel initially clears the body. As the panel travels down the body, the aerodynamics then begin to push the windward panel closer to the vehicle, as expected. A comparison of predicted panel trajectories with and without the presence of the SLS body is provided below in Figure 1. As seen from the figure, ignoring the aerodynamic effect of the vehicle body (panel alone) results in a prediction of the panel trajectory which is significantly closer to the body.

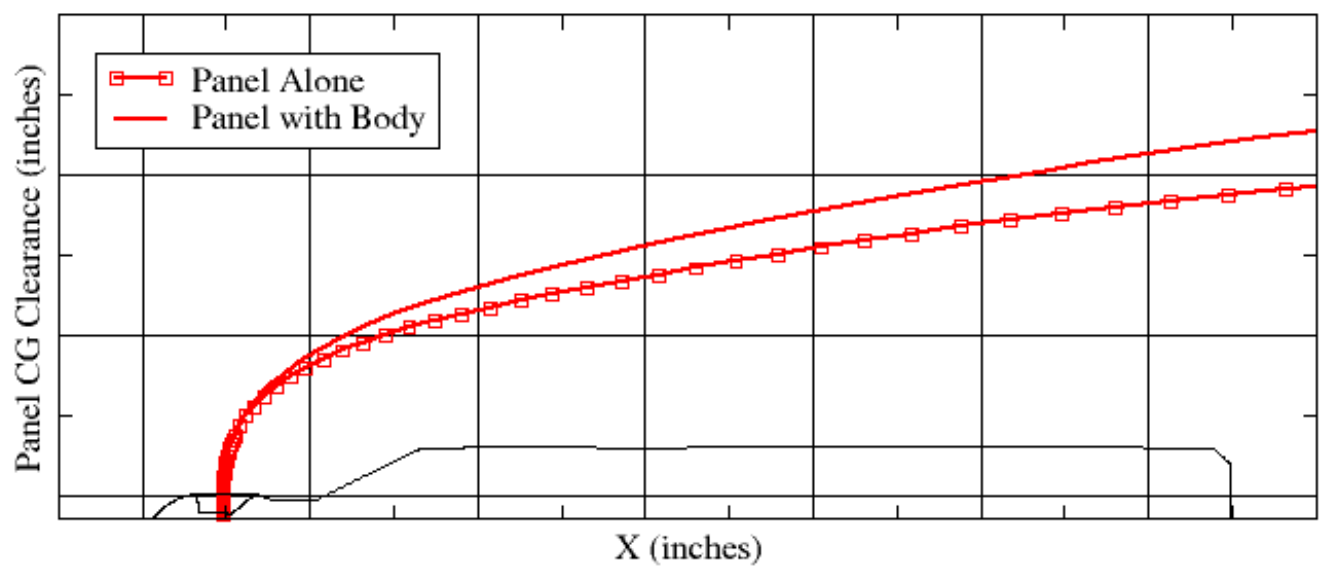

Figure 1 Panel Trajectory Predictions: Panel alone vs Panel with Inclusion of SLS Body

The primary goal of the SM panel jettison database is to create a tool which can be coupled with any 6-degree-offreedom (DOF) dynamics model to rapidly predict SM panel separation from the SLS vehicle. Development of the database requires CFD analysis of the SLS vehicle and SM panels in various relative orientations consistent with SM panel flight space and in a proper free stream environment. The resulting database is divided into three zones (see Figure 2):

- Panels on the hinge during initial separation. Both vehicle and panel-to-panel aerodynamic effects are included (orange).

- Panels in near proximity to the vehicle. Vehicle aerodynamic effects are included but panel-to-panel effects are neglected (green).

- Panels alone in the freestream environment with no vehicle interference effects (red).

Each of the three zones is discussed in the following sections. In these sections, the method for obtaining the data along with a brief discussion of the data is given. The resulting data from the CFD analysis is implemented into a MATLAB ${ }^{\circledR}$ code which allows the user to query the database and interpolate between points in the database. A brief discussion of the MATLAB ${ }^{\circledR}$ code is also presented. The final section shows results comparing the interpolated aerodynamic data versus a loosely-coupled approach which directly utilizes the CFD solver in the 6DOF process. 


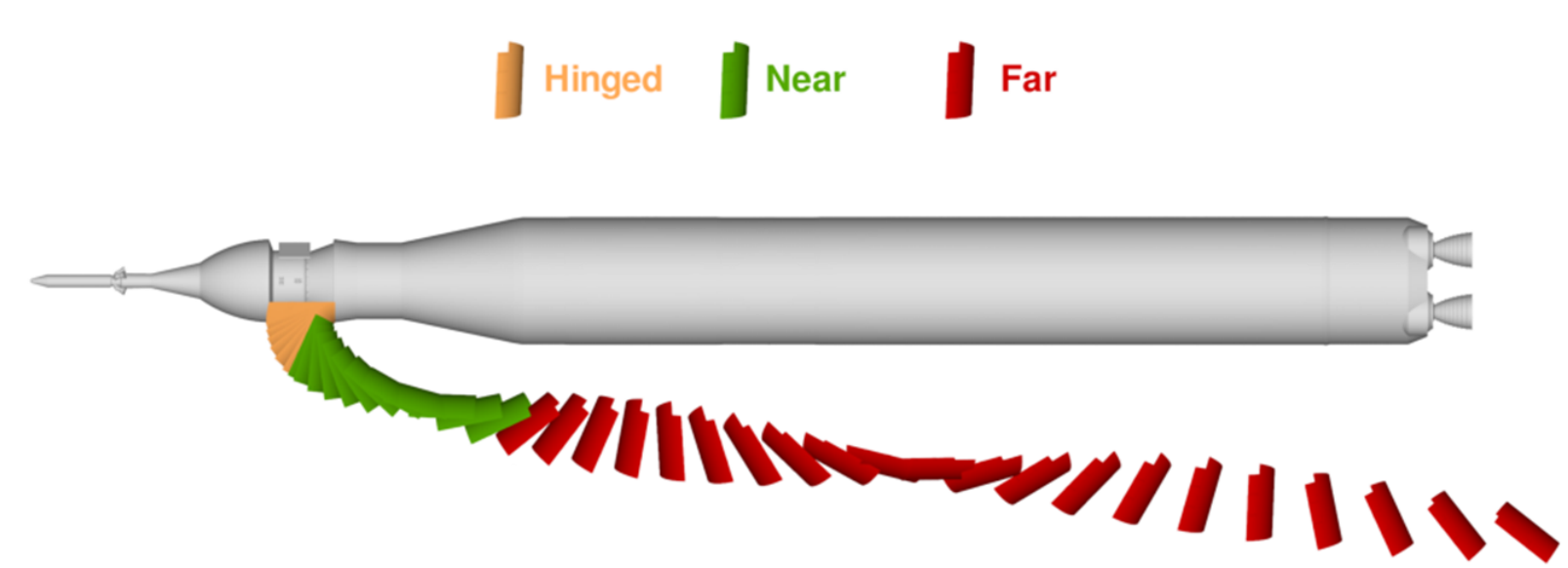

Panel 3, $\alpha=-18, \beta=0$

Not all hinged cases shown

Figure 2: Displays the three database zones, attached (orange), near proximity (green), and far/alone (red).

The SM panel geometry is shown in Figure 3. Due to restrictions the true dimensions are not available in this paper. The panel clocking is shown in Figure 4. All of the aerodynamic coefficients provided in the database are non-dimensionalized by the area and length of the panel. The area of the panel is the projected area computed by multiplying the length and the width of the panel.

All of the CFD simulations provided in the database were obtained using the FlowCart/Cart3D adaptive Cartesian Euler solver developed by NASA Ames ${ }^{5,6}$. The adjoint adaptation capabilities of Cart3D were used for each of the three zones, though the number of adaptations varied between each zone. For all three zones, the adaptation functional was a composite of the force components on each of the three panels, equally weighted. The goal was to obtain meshes in excess of 3 million cells for each simulation. Depending on the panel orientations and proximity to the body, some meshes exceeded 6 million cells. 


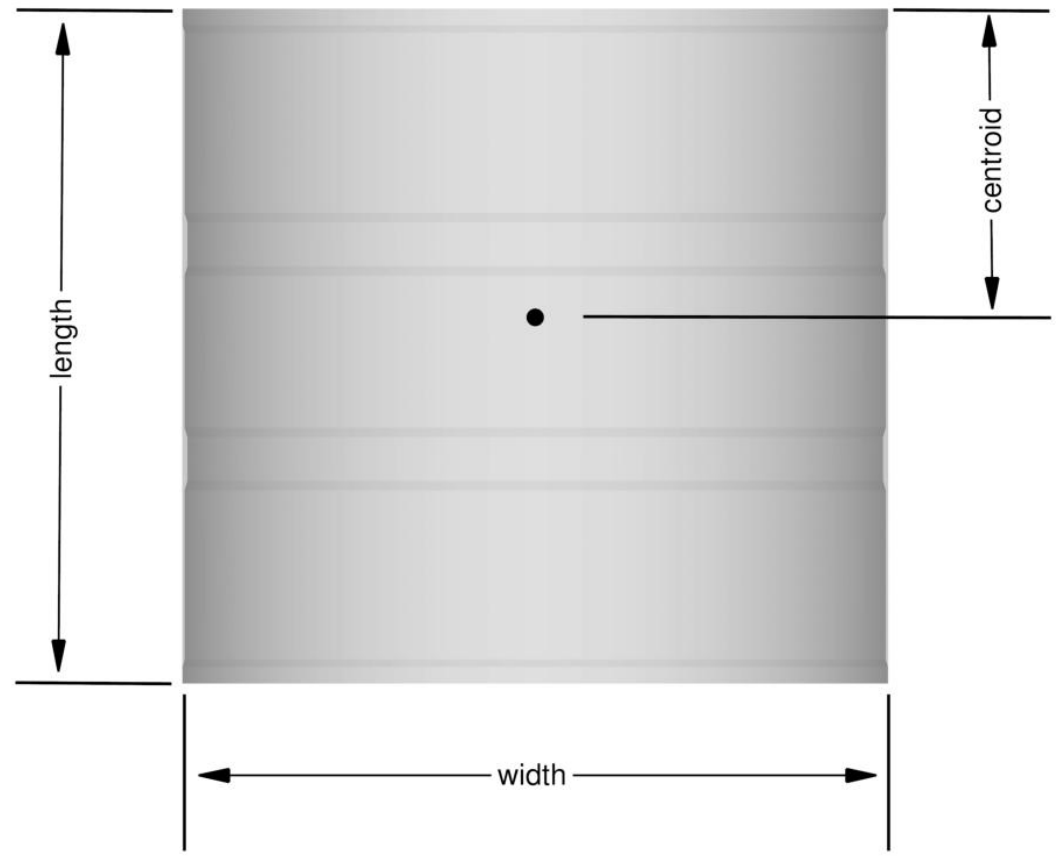

a. Top View

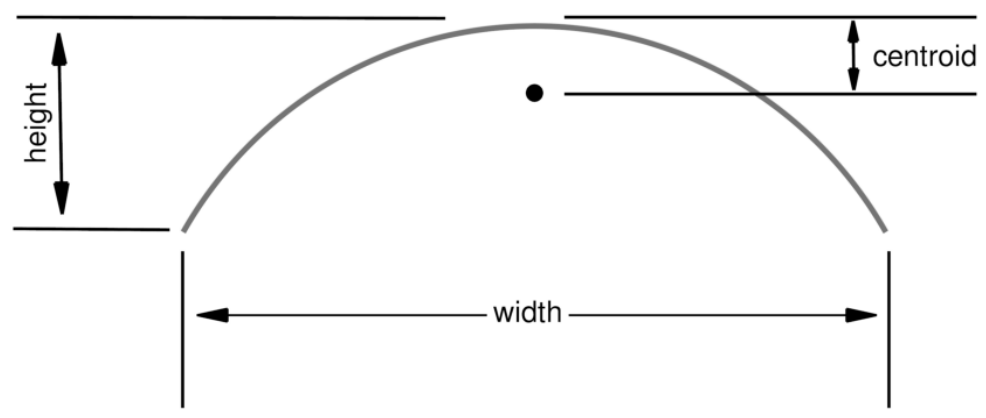

b. Front View

Figure 3: Panel Geometry 


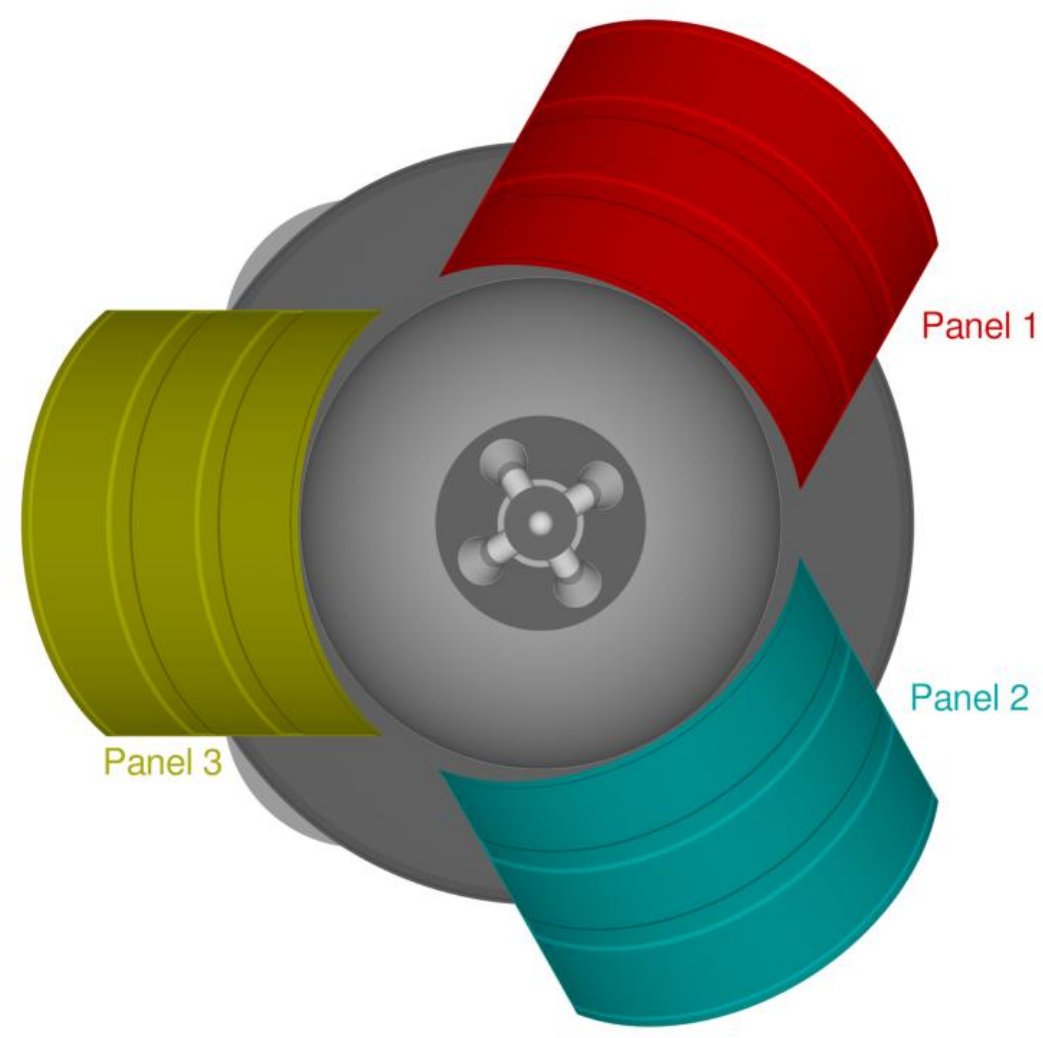

Figure 4: Panel clocking around SLS core (View from front to aft).

NASA Guidance, Navigation and Control (GNC) provided bounding trajectory points for the database. Table 1 shows the bounding cases provided by GNC. In developing the database, two choices were considered:

1. Construct the database in a traditional manner, bounded by angle of attack and side slip. Due to time constraints, a single Mach number would be chosen. It was determined that the single Mach number would split the bounds provided by GNC; namely Mach $=7.0$.

2. Produce a database with associated angle of attack and side slip tied to the Mach number and simulate the results at the boundary end of Mach numbers; approximately 5.5 and 8.5 respectively. For the same number of stations in time, this requires exactly half the number of simulations.

In order to quantify the effects of both choices, a number of CFD simulations were obtained. The cases examined were:

- Panels open at 30 degrees, Mach 5.4, alpha -21 , beta -5 and +4

- Panels open at 60 degrees, Mach 5.4, alpha -21 , beta -5 and +4

- Panels open at 30 degrees, Mach 8.55, alpha -21 , beta -5 and +4

- Panels open at 60 degrees, Mach 8.55, alpha -21 , beta -5 and +4

The results showed that the most significant Mach number effect (between Mach 5.4 and Mach 8.55) occurred for Panel 2 (the most windward panel) at the 30 degree panel rotation angle and produced a moment difference of $5.9 \%$. The maximum side-slip effect occurred on Panel 2 at the 60 degree rotation angle and also produced a moment difference of approximately 5.9\%. The results showed that either approach would result in a database of similar accuracy. The option of having the angle of attack and side slip determined as a linear function of chosen Mach number seemed like an overly restrictive element of the database. Furthermore, this limitation could possibly 
cause the database to lose relevance if dispersed conditions change. Therefore it was determined that the larger computational matrix provided the most flexibility. Table 2 provides the Mach number, angle of attack, and side slip combinations used to create the aerodynamic coefficient database.

Table 1: Bounding trajectory points provided by GNC.

\begin{tabular}{||l|c|c|c|c|c|c|c|c||}
\hline Mach number & 5.99 & 5.53 & 5.85 & 5.9 & 8.55 & 7.45 & 8.48 & 8.32 \\
\hline Angle-of-attack $(\boldsymbol{\alpha}), \mathbf{d e g}$ & -20.6 & -20.75 & -20.85 & -20.43 & -15.92 & -15.01 & -15.81 & -15.09 \\
\hline Sideslip angle $(\boldsymbol{\beta}), \mathbf{d e g}$ & -4.51 & -4.78 & -4.77 & -5.01 & 3.6 & 3.72 & 3.45 & 3.21 \\
\hline
\end{tabular}

Table 2: Mach, angle of attack, and side slip angles for the computational database.

\begin{tabular}{|c|c|c|c|}
\hline Case \# & Mach & $\boldsymbol{\alpha}(\mathbf{d e g})$ & $\boldsymbol{\beta}(\mathbf{d e g})$ \\
\hline 1 & 7.0 & -21 & -5 \\
\hline 2 & 7.0 & -21 & 5 \\
\hline 3 & 7.0 & -15 & -5 \\
\hline 4 & 7.0 & -15 & 5 \\
\hline
\end{tabular}

\section{Aerodynamic Database}

\section{A. Hinged Data}

This section discusses the CFD data for panels on the hinge during initial separation where not only the vehicle, but the panel-to-panel aerodynamic effects are important. The database assumes the panel will remain hinged until $60 \pm 5$ degrees of rotation. The goal is to provide 6-DOF forces and moments for use when the panel rotation is constrained about the hinge. The aerodynamic forces and moments are provided for each of the conditions listed in Table 2. The geometric matrix consists of:

- Panels rotate from 0 to 65 degrees by 5 degree increments

- A maximum of 15 degrees difference is allowed between any two panels

- For 0, 5, and 10 degrees, the panels are not allowed to vary from each other.

The last geometric constraint results from the SLS torsional spring design. In the current design, the spring operates over more than 10 degrees of rotation. During this time, the panels are dominated by spring forces and will not vary significantly over the first 10 degrees of travel. The geometric matrix yields a total of 362 CFD simulations per aerodynamic condition. The four aerodynamic conditions and 362 geometric conditions result in a total of 1,448 simulations for the hinged panel dataset. The reference parameters are the panel area and length. In addition, the moment reference point for these simulations is the hinge axis. Therefore, the moment coefficients provided in the database are the aerodynamic hinge moments.

Figure 5 shows the hinge moment coefficients on Panel 2 for the four aerodynamic conditions. Every data point for Panel 2 is shown in the figures. The spread of the data for a given panel angle is due to the influence of Panel 1 and Panel 3 on Panel 2. The red line shows the data from a similar study ${ }^{1}$ for the Ares launch vehicle. The Ares data was obtained on the A104 configuration at Mach 6.5, $\alpha=-13$, and $\beta=0$. The Ares study did not allow panels to be at different angles. The Ares data are a relevant comparison and provides some verification of predictions as 
the panel configurations and the forward section of the Ares vehicle are similar to SLS, as well as the panel separation flight conditions.

In Figure 5, at each panel angle, there are 37 potential orientations of the other two panels. The best match to the Ares data is shown in Figure 5(c), which corresponds to Case 3. Case 3 angle of attack (-15) is closest to the Ares angle of attack (-13). Note from Figure 5, as the angle of attack decreases, the hinge moment increases for a given side slip angle. Also note as the panel angle increases, the influence of the other panels decreases, becoming a very narrow band at high panel angles. As a result, panel-to-panel effects were considered small and ignored after the panels were released from the hinge. Panel 1 and Panel 3 figures are not presented here. Panel 2 (windward) is the most likely to re-contact the surface of the vehicle, and therefore the focus of the paper.

Twelve viscous check cases were performed to determine how well the inviscid CFD solver captures the physics important for SM Panel separation. The viscous simulations were performed using the Loci/Chem unstructured flow solver ${ }^{7,8}$. The results for panel 2 are shown in Table 3 . The results show good agreement with the average difference of $4.9 \%$ over the twelve cases with most cases falling well below the $4.9 \%$ average. There is a single outlier in the cases examined occurring when all three panels are at 15 degrees rotation, where the difference between inviscid and viscous predicted hinge moment is $21.1 \%$. This outlier does not decrease confidence in results for two reasons. First, this is but a single orientation at a transient point in time which may or may not actually occur and if so, only occurs for a brief moment. Secondly, and more importantly, the motion of the panels at 15 degrees hinge-rotation orientation is still dominated by the spring force used to initiate panel separation. As the panels rotate further on the hinge, the aerodynamics become significantly more important for trajectory determination and integrated results between viscous and inviscid solutions show much closer agreement. For the panel of interest, good agreement between inviscid and viscous predictive techniques is obtained due to physics of the hypersonic flow experienced by the windward panel. In the more leeward panels which experience significant flow separation, larger deviations between inviscid and viscous results occur.

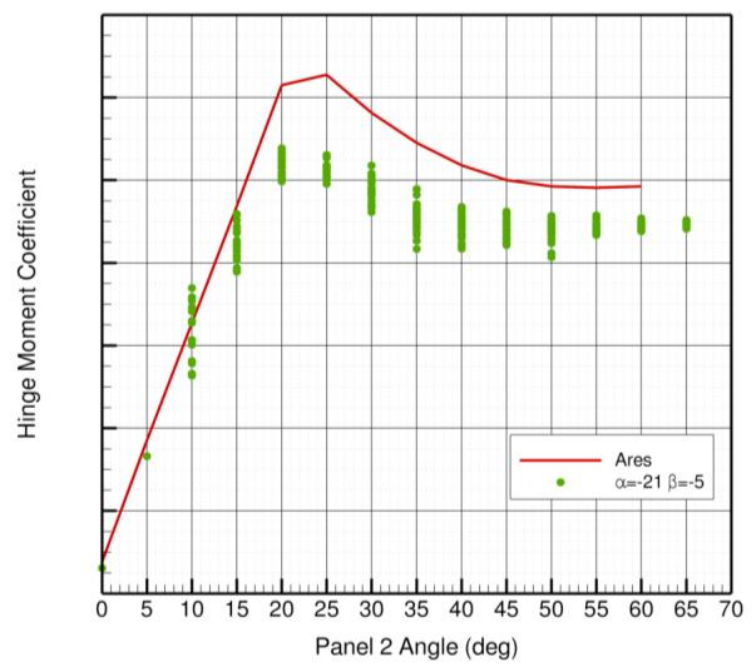

(a) Case 1 (Mach 7.0, $\alpha=-21, \beta=-5$ )

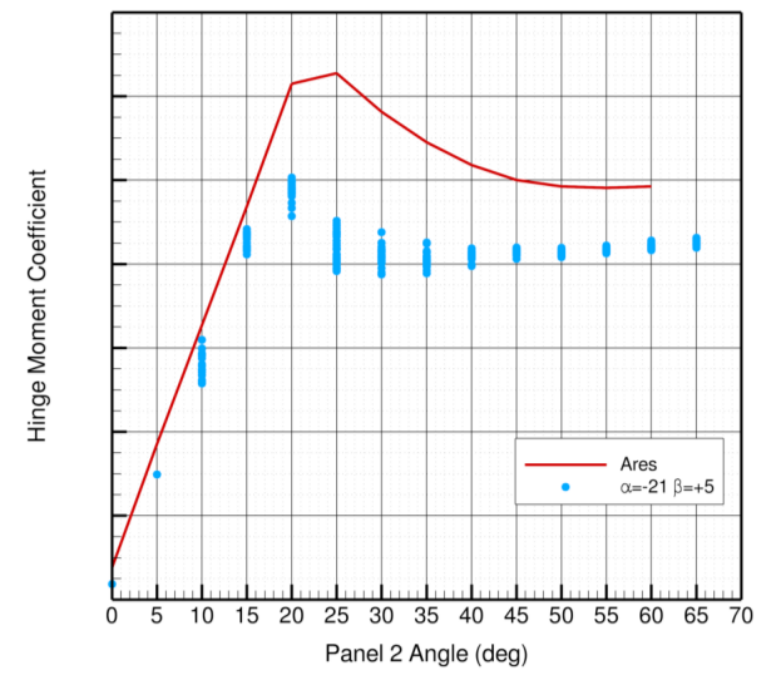

(b) Case 2 (Mach 7.0, $\alpha=-21, \beta=+5)$ 


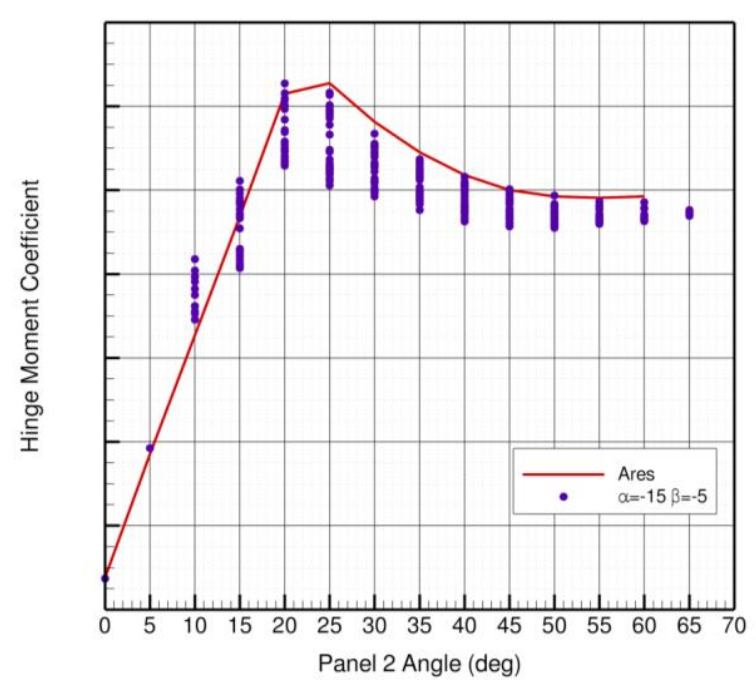

(c) Case $3($ Mach $=7.0, \alpha=-15, \beta=-5)$

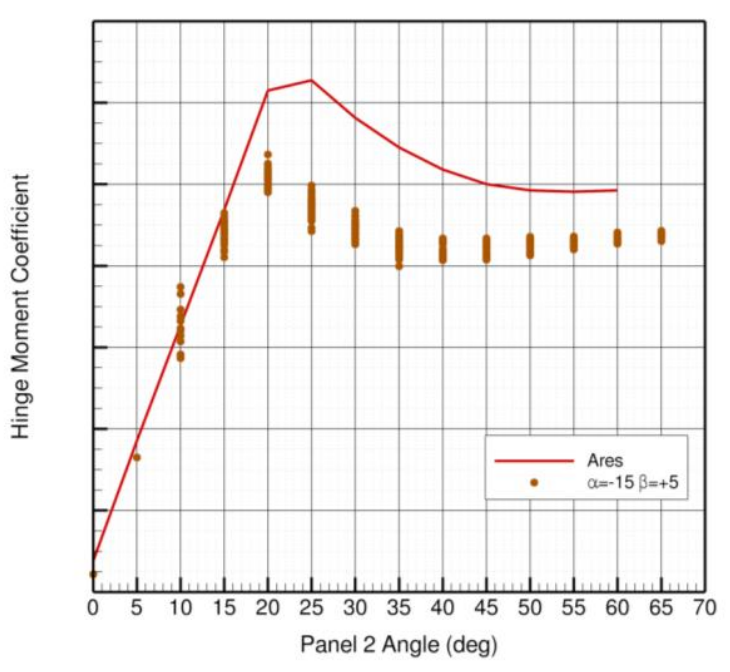

(d) Case $4($ Mach $=7.0, \alpha=-15, \beta=+5)$

Figure 5: Panel 2 hinge moment coefficient for the four aerodynamic conditions.

Table 3: Hinged data viscous comparison for panel 2.

\begin{tabular}{|c|c|c|}
\hline Condition & $\begin{array}{c}\text { Orientation } \\
\text { (P1-P2-P3) }\end{array}$ & \% Difference \\
\hline$\alpha=-21 \beta=-5$ & $30-30-30$ & 8.1 \\
\hline$\alpha=-21 \beta=+5$ & $30-30-30$ & 0.6 \\
\hline$\alpha=-15 \beta=-5$ & $30-30-30$ & 2.3 \\
\hline$\alpha=-15 \beta=+5$ & $30-30-30$ & 3.4 \\
\hline$\alpha=-21 \beta=-5$ & $45-45-45$ & 3.7 \\
\hline$\alpha=-21 \beta=+5$ & $45-45-45$ & 1.4 \\
\hline$\alpha=-15 \beta=-5$ & $45-45-45$ & 7.3 \\
\hline$\alpha=-15 \beta=+5$ & $45-45-45$ & 0.6 \\
\hline$\alpha=-15 \beta=-5$ & $15-15-15$ & 21.1 \\
\hline$\alpha=-21 \beta=-5$ & $35-40-25$ & 4.5 \\
\hline$\alpha=-21 \beta=+5$ & $45-50-55$ & 3.1 \\
\hline$\alpha=-15 \beta=+5$ & $55-60-45$ & 2.7 \\
\hline
\end{tabular}

\section{B. Near Proximity Data}

This section discusses the CFD data for panels in the near proximity zone in which the SLS vehicle aerodynamic effects could still be considered important. The goal is to provide 6-DOF forces and moments for use when the panel is off the hinge, but still under the influence of the vehicle body. The aerodynamic forces and moments are provided for each of the aerodynamic conditions listed in Table 2. The geometric matrix consists of: 
- Six panel stations, 3 axial stations and 2 radial stations at each axial station

- Pitch \pm 45 degrees from the baseline orientation in 15 degree increments

- Roll \pm 10 degrees from the baseline orientation in 10 degree increments

- Yaw \pm 10 degrees from the baseline orientation in 10 degree increments

- Rotation order is pitch, roll, yaw

Each of the 6 stations has a deflection matrix of 63 simulations (pitch, roll, and yaw). The geometric matrix consists of a total 378 simulations. Combined with the four aerodynamic conditions, the total near proximity matrix contains 1,512 simulations. Figure 6 shows the axial and radial definitions of the 6 stations along with their baseline orientations. The baseline stations were determined from an average of four Cart3D/6-DOF simulations for each of the aerodynamic conditions given in Table 2. Stations S11, S21, and S31 are the average Cart3D/6-DOF orientations. Three additional stations were included at increased radial distance of half the panel length from the center line. These are stations S12, S22, and S32.

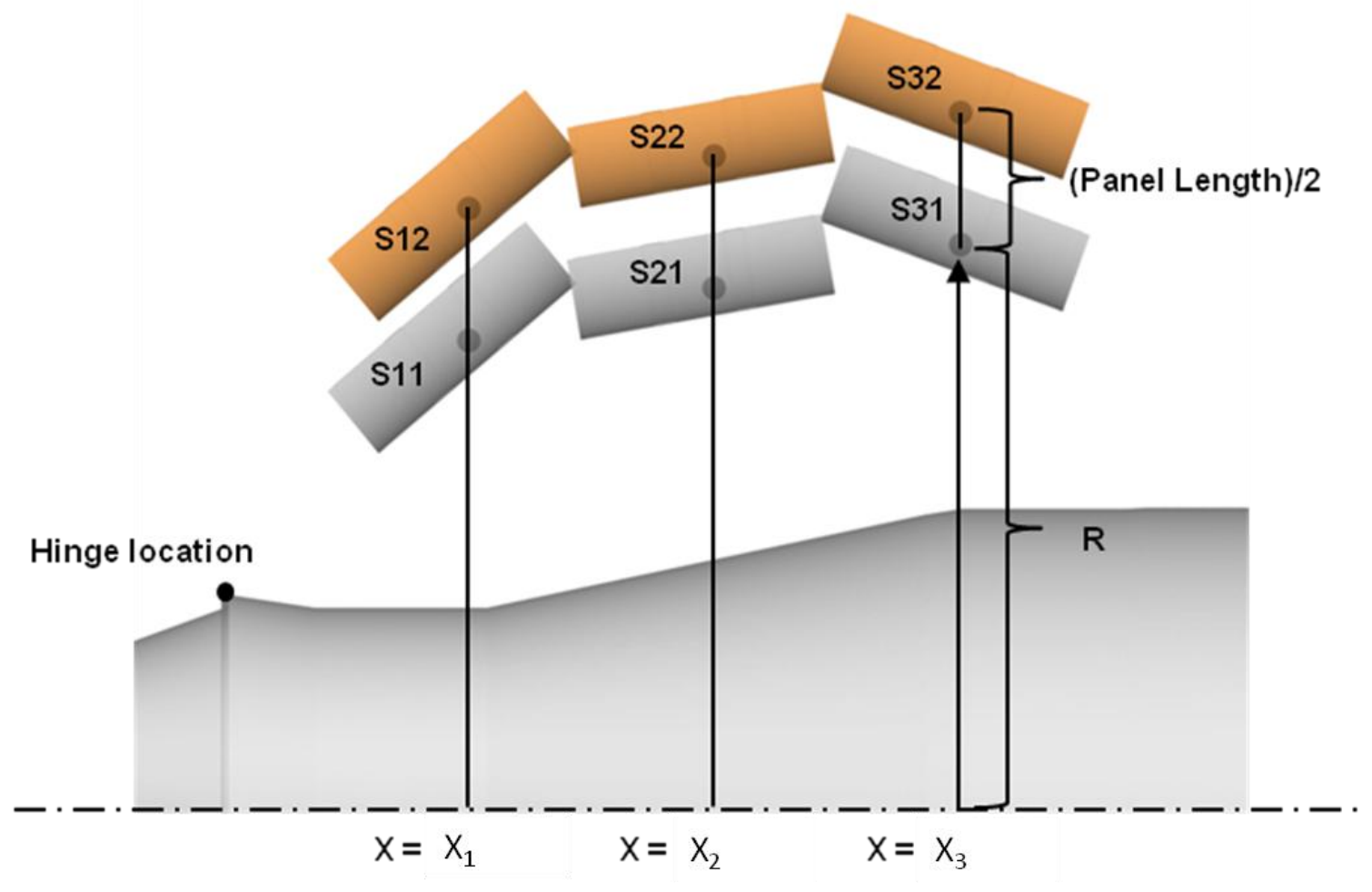

Figure 6: Near proximity axial and radial station definitions.

Figure 7 shows the perturbations of the panel angles about the baseline for each station. Figure 7a shows all of the pitch perturbations for one of the stations. The baseline orientation is shown in pink with the perturbations shown in yellow. The pitch varies from \pm 45 degrees from the baseline in increments of 15 degrees. Figure $7 \mathrm{~b}$ shows all of the roll perturbations for one of the stations. The pink panel in Figure $7 \mathrm{~b}$ is the baseline orientation, the yellow is the +10 degree roll, and the blue panel is the -10 degree roll. Figure $7 \mathrm{c}$ shows the same information for the yaw. The reference parameters are the panel area and length. The moment reference point is the centroid of the panel. Note that the \pm 10 degree roll and yaw perturbations encompassed all of the roll/yaw orientations predicted by the Cart3D/6-DOF simulations.

Due to the amount of data available, only a small snapshot of the data is presented here. Figure 8 shows Cfx versus pitch angle for panel 2 at station S11. Figure 9 shows Cfy versus pitch angle for panel 2 at station S11. Figure 10 shows Cfz versus pitch angle for panel 2 at station S11. Note that every data point for Panel 2 is shown in these figures. The spread of the data for a given panel pitch angle is due to the different freestream conditions and the various roll and yaw orientations. 
It was of interest to understand how much influence the "near proximity" region of the database had on panel trajectory. Subsequent panel trajectory predictions were made ignoring the proximity region of the database, retrieving aerodynamic coefficients from the panel-alone data after panel release from the hinge. Those results, while not shown in this paper, show for the flight conditions examined in this analysis, the proximity region of the database provides only a second order effect on trajectory. However, the proximity region of the database was still provided as it does provide a higher fidelity answer and primarily due to the possibility that future examined flight conditions might orient the panel relative to the body such that results in this region have a more significant impact on trajectory.

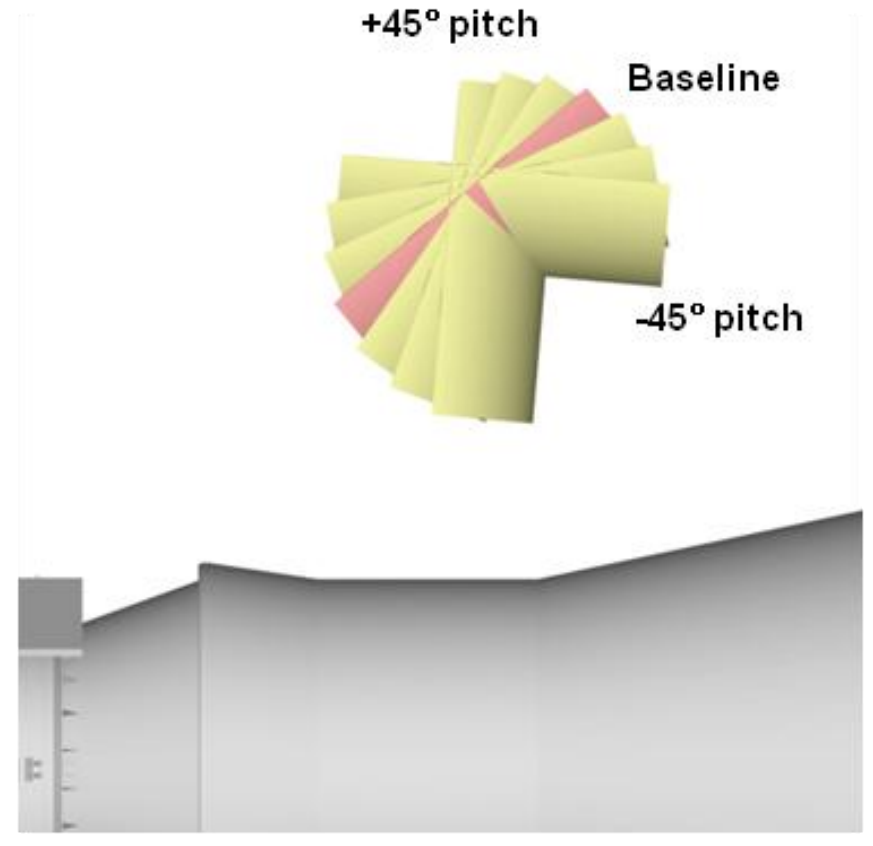

Subfigure 6a: Pitch angle deflections
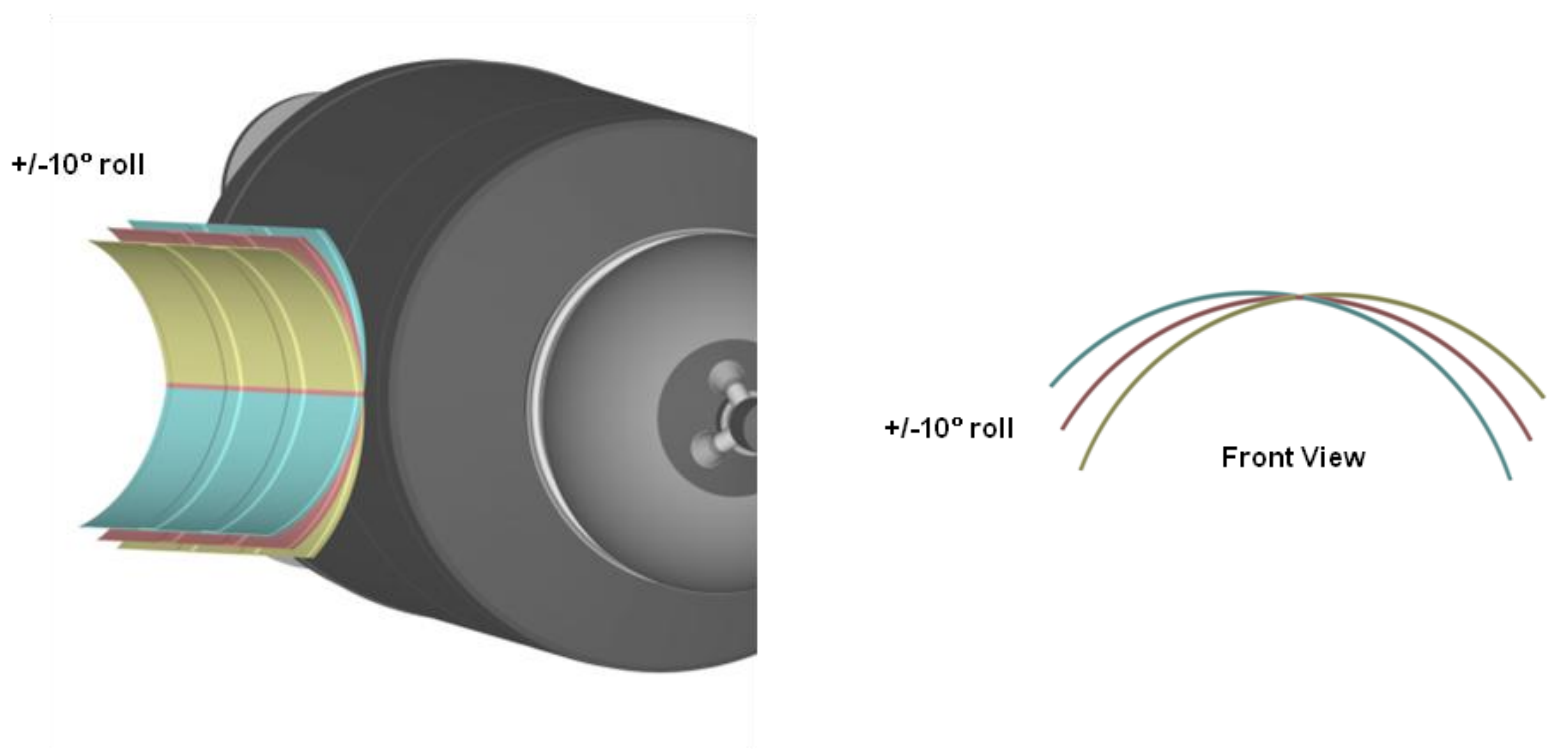
Subfigure 6b: Roll angle deflections
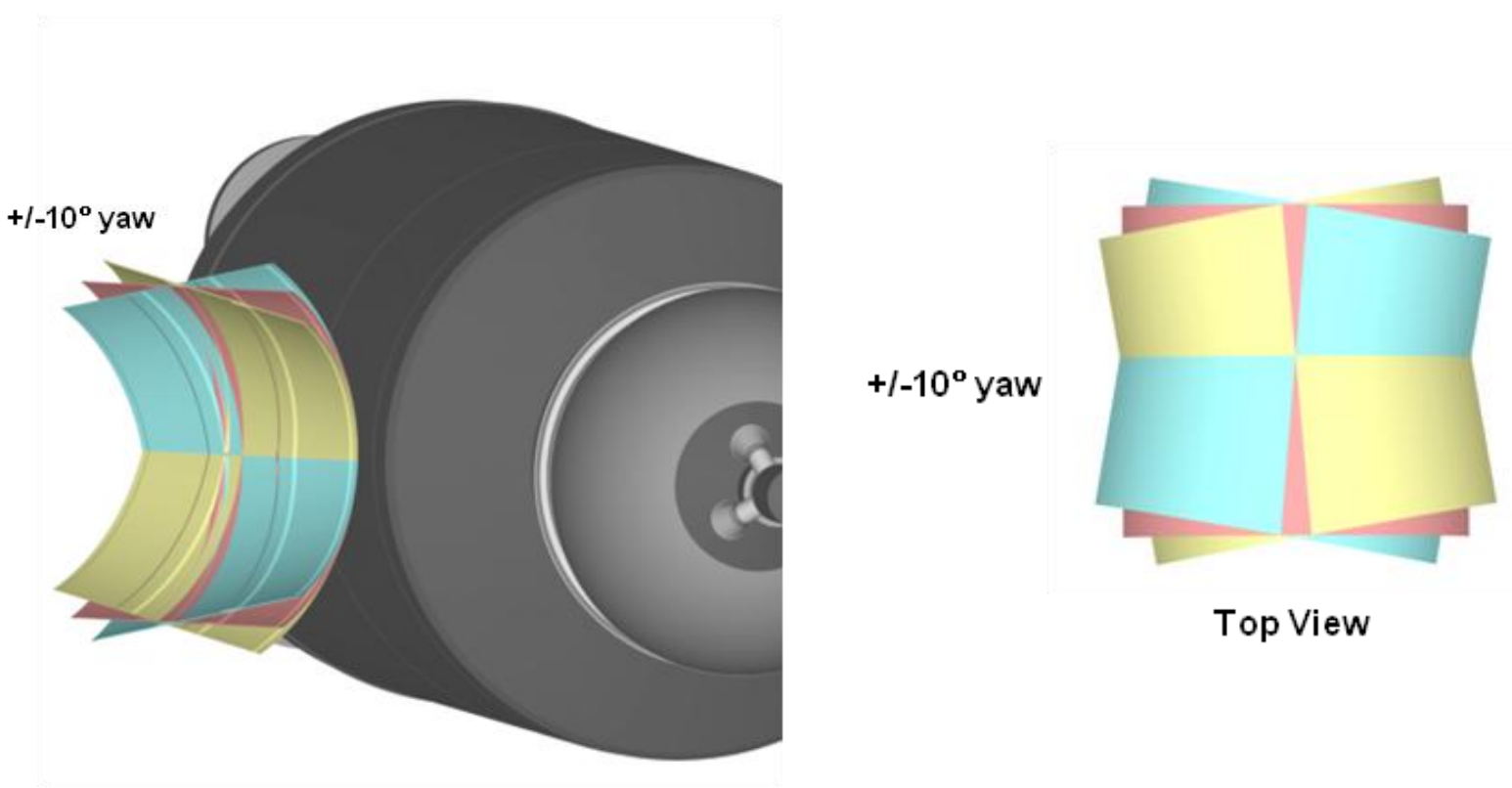

Top View

Subfigure 6c: Yaw angle deflections

Figure 7: Euler angle deflections from baseline for the near proximity cases.

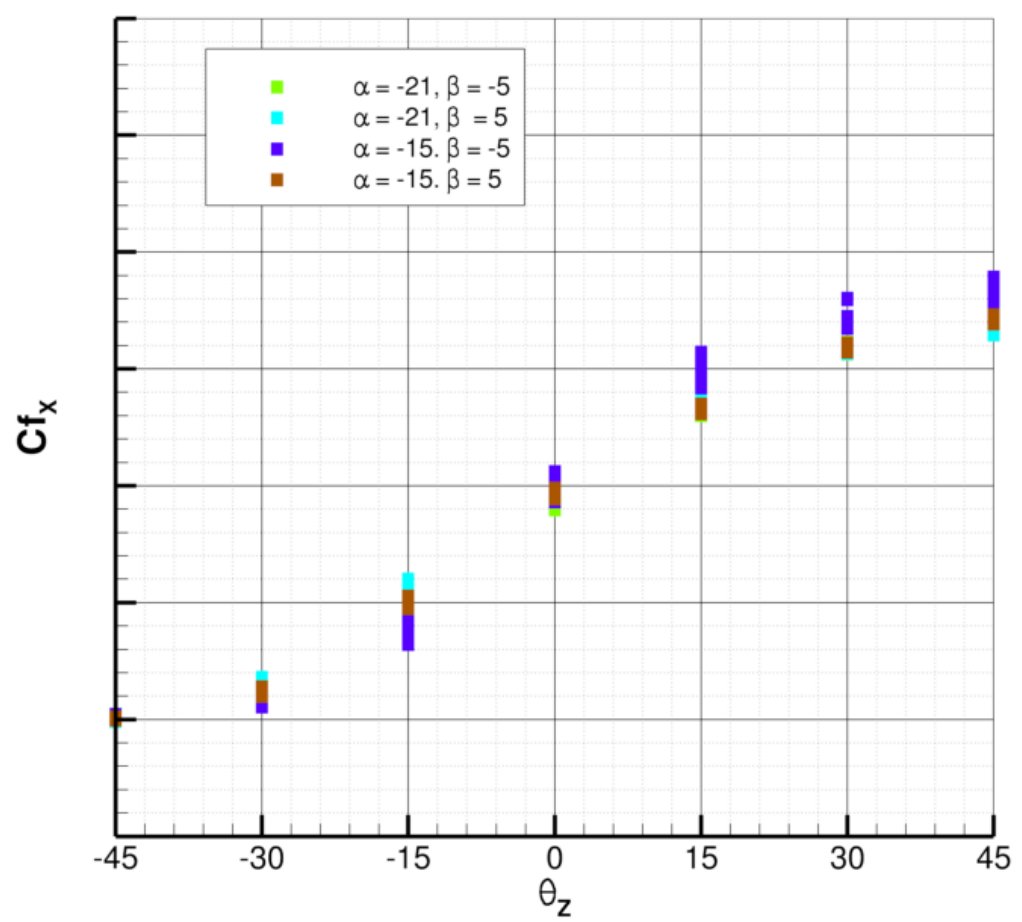

Figure 8: Panel $2 \mathrm{Cfx}$ versus pitch angle for station S11.

American Institute of Aeronautics and Astronautics 


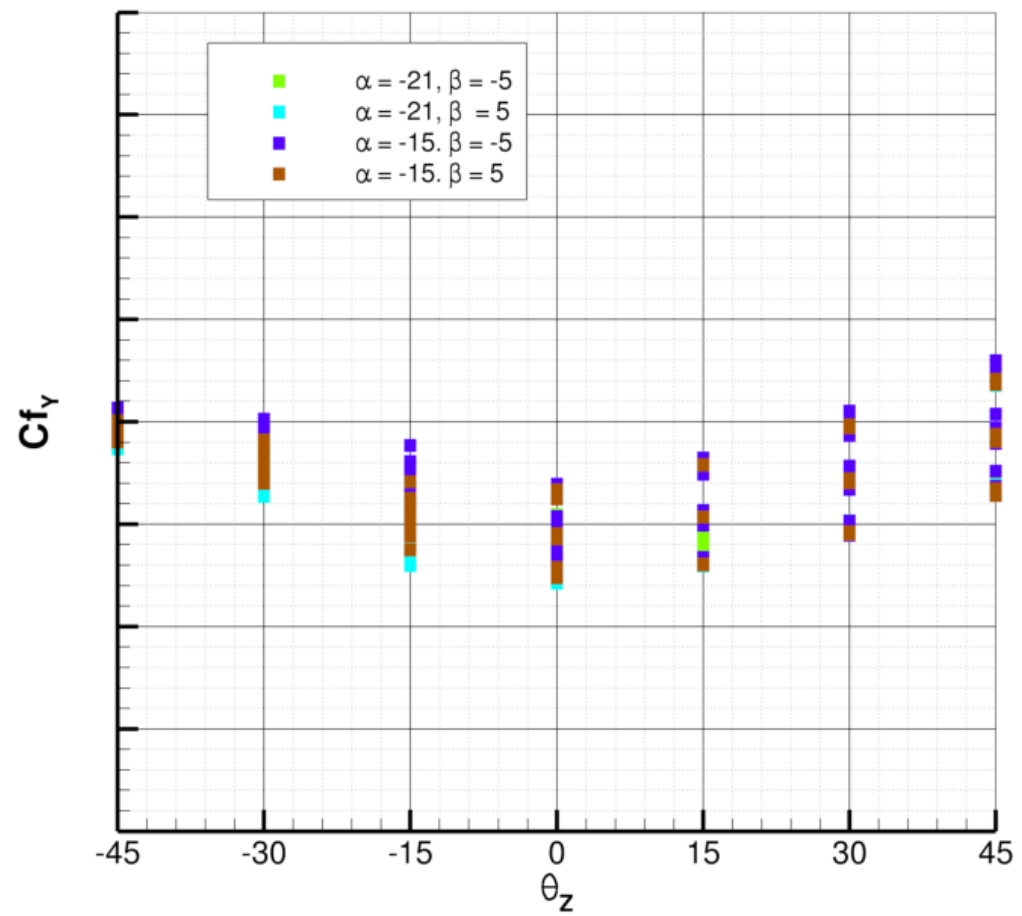

Figure 9: Panel 2 Cfy versus pitch angle for station S11.

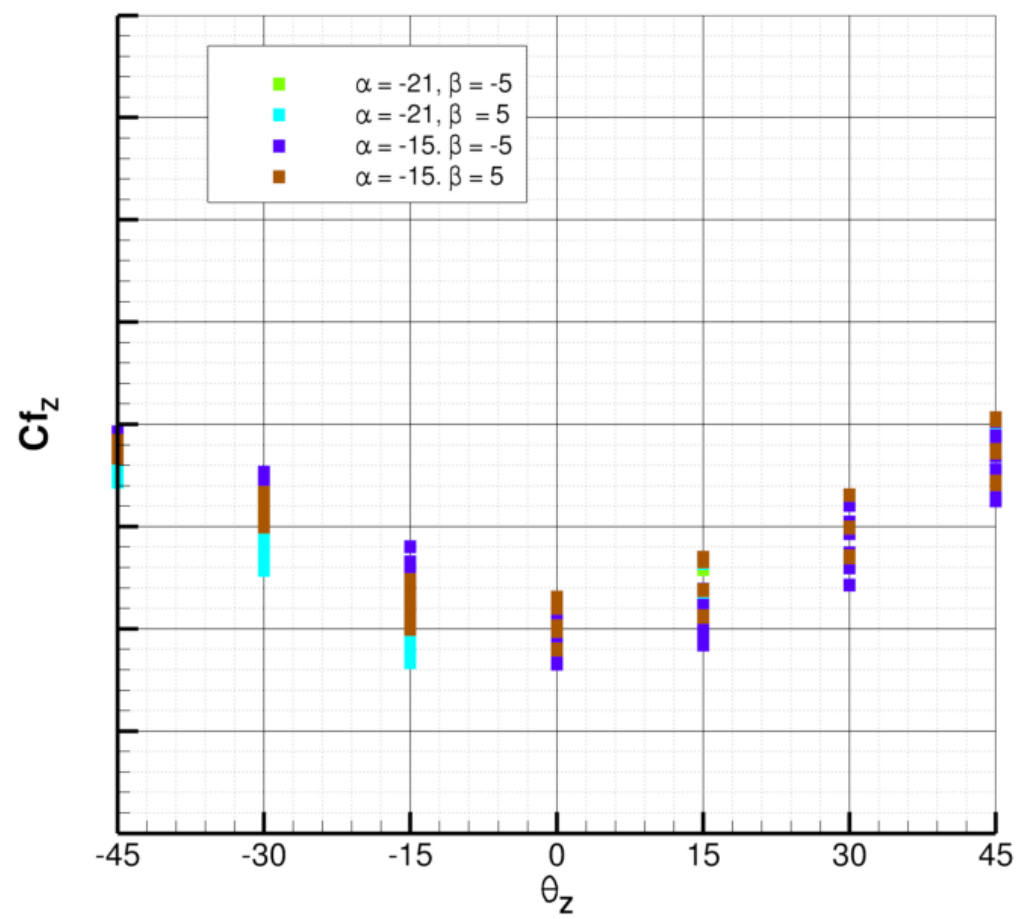

Figure 10: Panel $2 \mathrm{Cfz}$ versus pitch angle for stations S11. 


\section{Far (Panel Alone) Data}

This section discusses the CFD data for panels in a freestream flow without any influence from other panels or the vehicle body. The goal is to provide 6-DOF forces and moments for use when the panel is far enough from the vehicle that it does not influence the aerodynamic coefficients. Unlike the previous two zones, the data does not take into account vehicle angle of attack and side slip. To utilize the data in a 6-DOF simulation, the interpolation of the aerodynamic coefficients must be augmented for angle of attack and side slip. Figure 11 shows the orientation and coordinate system definition for the panel alone dataset. The origin of the coordinate system is located at the panel centroid (not shown in the figure). The geometric matrix for the panel alone dataset consists of:

- Rotation order: Z (pitch), X (roll), Y (yaw).

- Rotations are about the fixed X-Y-Z axis system shown in Figure 4.

- The Z-rotations (pitch) are from 0 to 345 by 15 degrees.

- The X-rotations (roll) are from 0 to 90 by 15 degrees.

- The Y-rotations (yaw) are from 0 to 180 by 15 degrees.

- The centroid of the panel is located at coordinate $(0,0,0)$.

- All rotations occur about the centroid of the panel.

The geometric matrix yields a total of 2,184 CFD simulations. There is only one aerodynamic condition, namely Mach $=7.0$, angle of attack $=0$, and side slip $=0$.

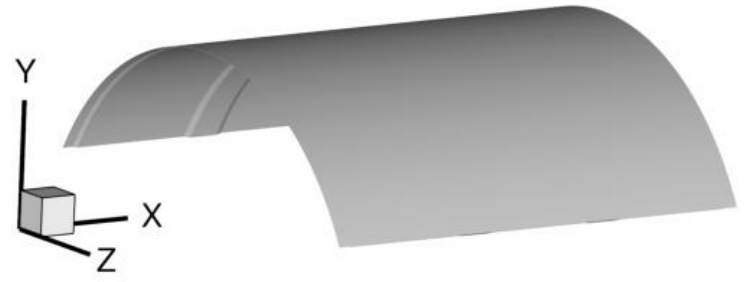

Figure 11: Panel alone coordinate system definition. Note, the origin of the coordinate system is at the centroid of the panel (not shown by the axis in the figure). The axes are for illustrative purposes only.

The reference parameters are the panel area and length. In addition, the moment reference point for these simulations is the centroid of the panel $(0,0,0)$. The CFD simulations are mirrored to create 360 degree coverage in the three rotation angles. This yields an aerodynamic coefficient matrix of 15,000 data points.

Figure 12 and Figure 13 show the normal force coefficient and pitching moment coefficient for the panel alone dataset. The figures compare the results for the SLS database versus the Ares A104 database. Note, the coefficients shown in both figures are in the body fixed coordinate system, which is not the coordinate system used for the SLS panel alone database. While the panel geometry is similar between SLS and Ares, there are differences that exist in the solutions. The Ares A104 database was performed without the solution adaptive capabilities of Cart3D, while the SLS database took full advantage of the adjoint capability of Cart3D. In addition, the Mach number for the Ares database was 6.5 and the Mach number for the SLS database is 7.0. 


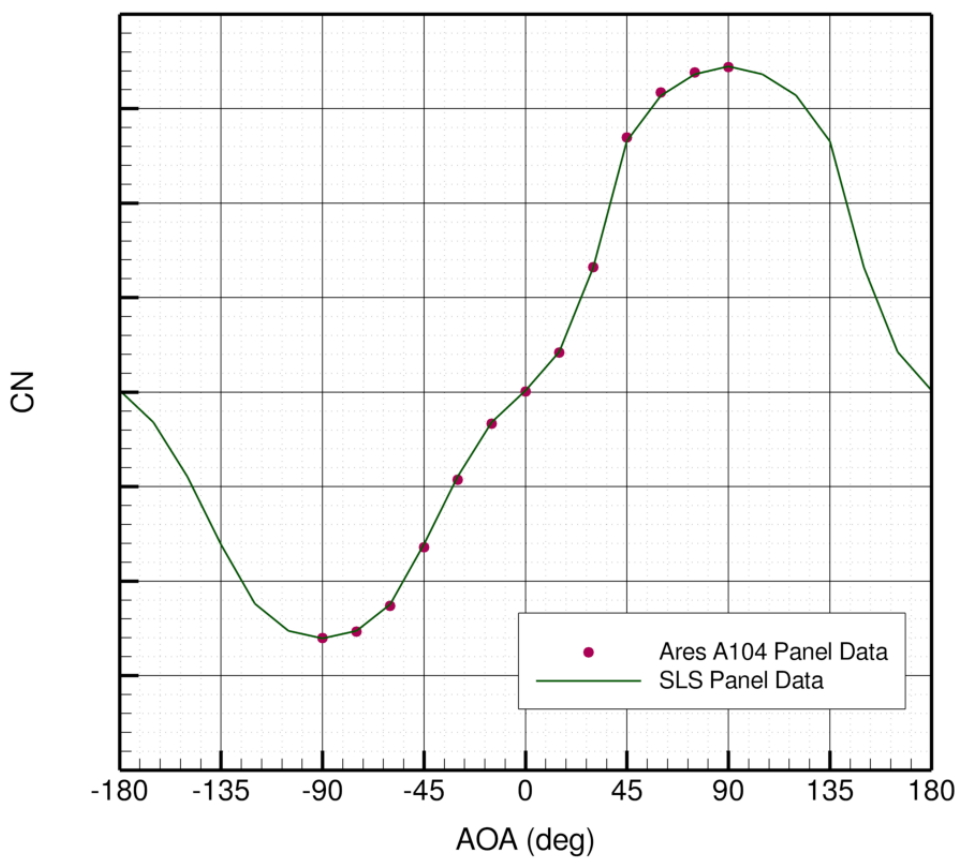

Figure 12: Comparison of normal force coefficient for SLS panel alone data and Ares A104 panel alone data. The force coefficient shown is in the body-fixed coordinate system. Angle of attack is equivalent to the pitch angle.

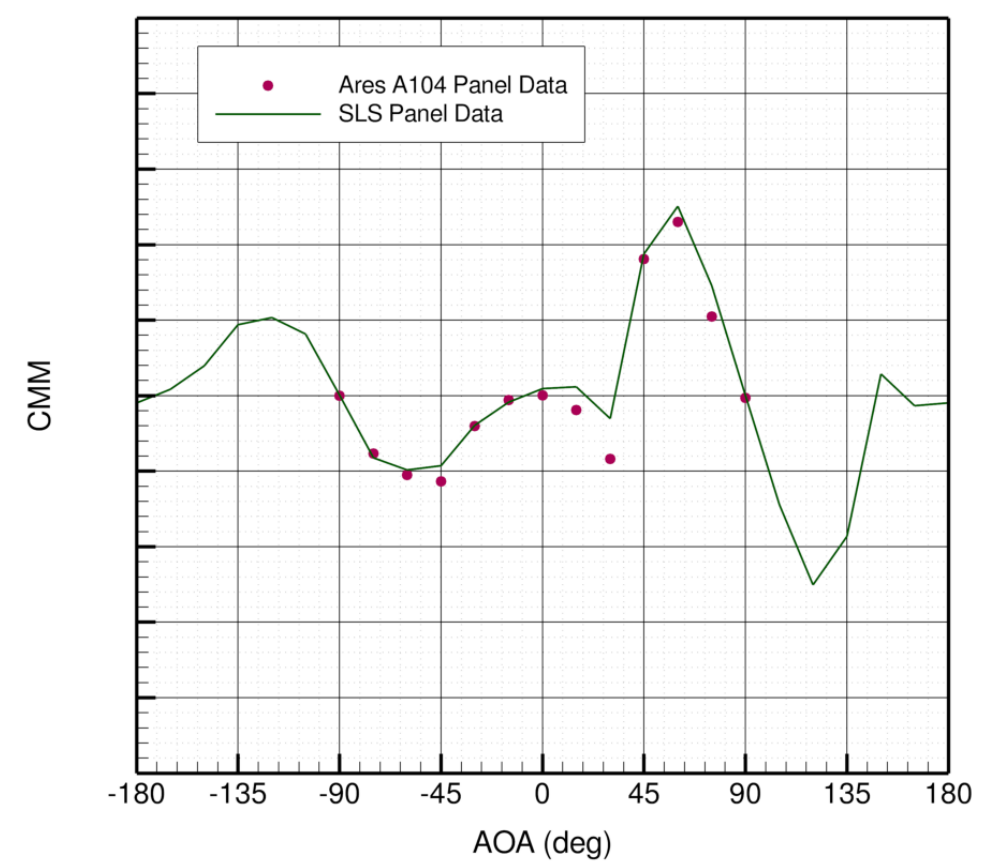

Figure 13: Comparison of pitching moment coefficient for SLS panel alone data and Ares A104 panel alone data. The moment coefficient shown is in the body-fixed coordinate system. Angle of attack is equivalent to the pitch angle. 


\section{MATLAB ${ }^{\circledR}$ Scripts}

A "black box" MATLAB ${ }^{\circledR}$ code was written to query the CFD data at input values of vehicle/SM panel parameters and return the aerodynamic force and moment coefficients of the SM panels as they are jettisoned from the vehicle. While the CFD data fully defines the aerodynamics of the SM panel jettison event, the MATLAB ${ }^{\circledR}$ code provides an easy, accurate "black box" to obtain the aerodynamic coefficients for each of the panels given the multivariate nature of the CFD data. Just as the CFD data was generated for three different regimes of flight for the SM panels, the MATLAB ${ }^{\circledR}$ functionality is divided into the three regimes shown in Figure 2. The code ensures the proper function for the relevant panel flight regime. The code also contains checks of the input vehicle/panel parameters against the bounds of the CFD data and provides a warning if the bounds of the CFD data are exceeded. Provisions have also been included in the coding to help ensure smooth transitions from one SM regime to the next with regards to the aerodynamic coefficients.

Figure 14 shows a flowchart of the general MATLAB ${ }^{\circledR}$ execution. Each of the three different flight regimes (hinged, near, and far) is dependent upon a differing number of independent variables. This necessitates a separate function for each zone. The main MATLAB $^{\circledR}$ function utilized for the interpolation/extrapolation of data was scatteredInterpolant. This function maps the dependent variable to a surface at given locations determined by the independent variables. The surface passes through the input values. This response surface can then be queried at any location to determine the dependent variable value at varying values of the independent variables. This function can perform both two- and three-dimensional interpolation. One benefit to using this function in this application is the input data need not be evenly distributed in the data space. For example, the angles of attack do not have to be evenly incremented, nor do they have to be the same for each data point. However, because scatteredInterpolant can at most perform three-dimensional interpolation and the data for two of the modeled zones are functions of more than three variables, there is a need to utilize scatteredInterpolant more than once.

Figure 15 shows a flowchart of the general execution of the attached (hinged) zone interpolation. The attached data is a function of five independent variables. Those are the angle of attack, the side slip angle, and the three panel deflection angles. Because the value of any given aerodynamic coefficient is a function of five independent variables, multiple calls to the scatteredInterpolant function are required. The first step of the algorithm builds a response surface for each of the four aerodynamic conditions $(\alpha, \beta)$ for each of the three panels. This step generates twelve response surfaces for each aerodynamic coefficient. The second step queries the response surfaces from step 1 using the user input panel deflections. For each panel this results in four values of each aerodynamic coefficient for each of the four aerodynamic conditions. These values are used to create an additional response surface that is a function of angle of attack and sideslip for each panel. This response surface is queried using the user input angle of attack and sideslip to arrive at our final aerodynamic coefficients.

The near zone is similar to the attached zone in that the aerodynamic coefficients are a function of three position angles of the panels as well as angle of attack and sideslip for each panel. The near zone has the additional independent variables of radial and axial distance. Because of this similarity the function to determine the aerodynamic coefficients for the near zone follows the same logic as that for the attach zone as outlined above. The difference is in that with the addition of the radial and axial variables more response surfaces need to be generated. In the first round of interpolation response surfaces are built using panel pitch, yaw, and roll angles as the independent variables for each value of angle of attack and sideslip per Table 2, as well as each value of radial and axial distance as shown in Figure 6, for each of the three panels. There is also one additional combination of axial and radial station which represents the panel immediately after release from the hinge. Thus, after querying each of the response surfaces there are twenty eight values for each aerodynamic coefficient for each of the three panels. Response surfaces are now built using angle of attack and angle of sideslip as the independent variables. After querying the surface at the desired values of angle of attack and sideslip this process yields seven aerodynamic coefficient values for each panel, one value for each station shown in Figure 6, plus the one extra station where the panel leaves the hinge. At this point these seven values are used to build a final response surface which is queried at the desired values of radial and axial location to determine the final aerodynamic coefficients for each of the three panels. Figure 16 shows a flowchart of the general execution of the near zone interpolation.

The far zone being a function of only three independent variables, pitch, yaw, and roll, requires only a single call to the scatteredInterpolant function. A response surface is built from the aerodynamic database data and can then be queried for any values of pitch, yaw, and roll that are input from the calling program. Figure 17 shows a flowchart of the general execution of the far zone interpolation. 


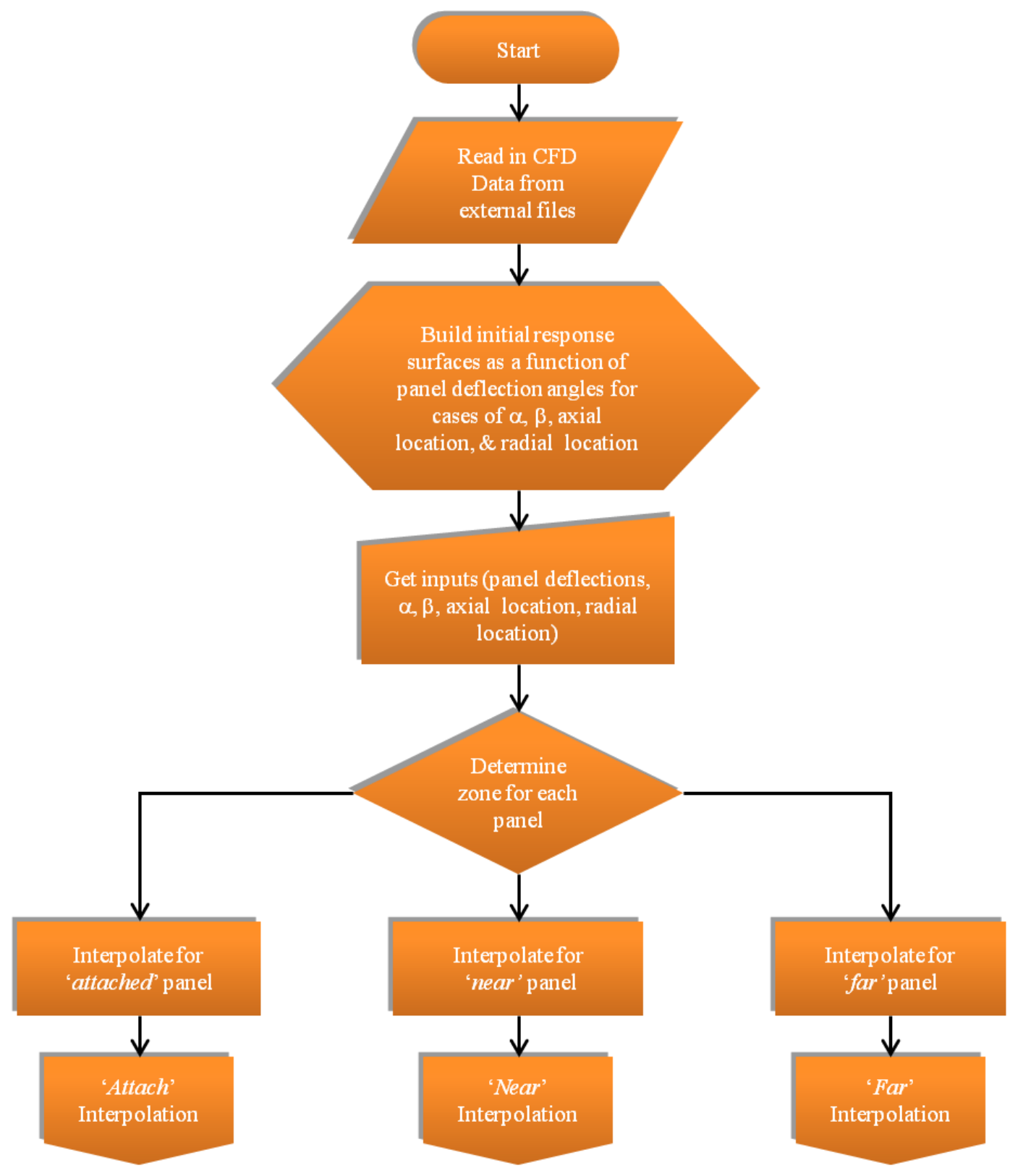

Figure 14: MATLAB ${ }^{\circledR}$ execution flowchart.

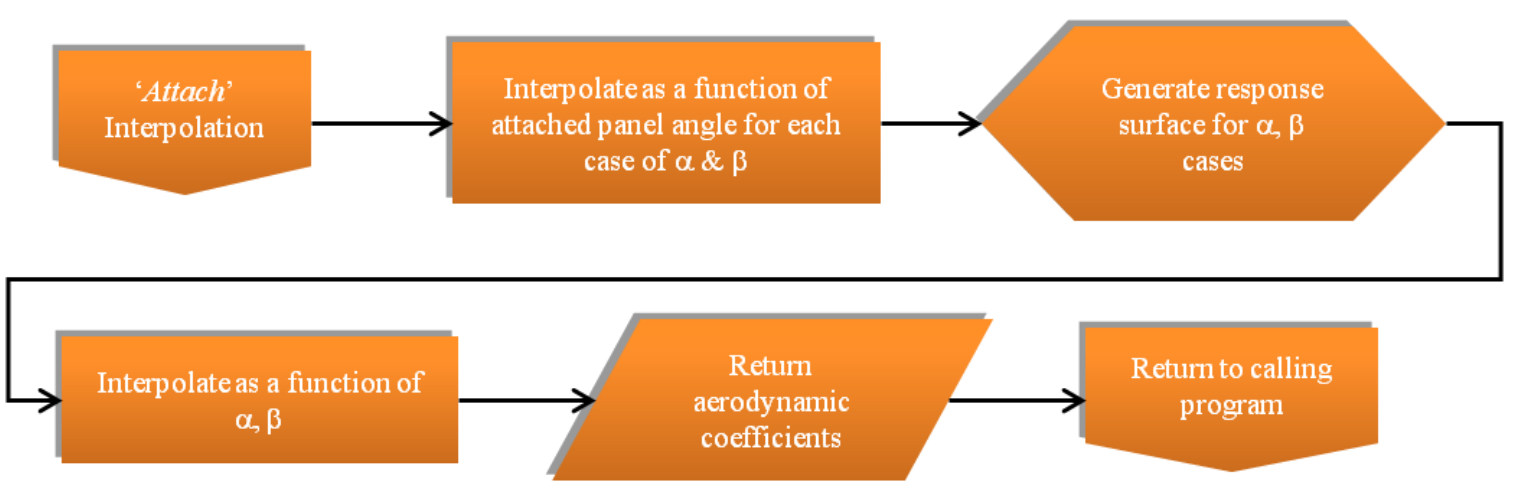

Figure 15: Flowchart of the attached panel interpolation execution.

American Institute of Aeronautics and Astronautics 


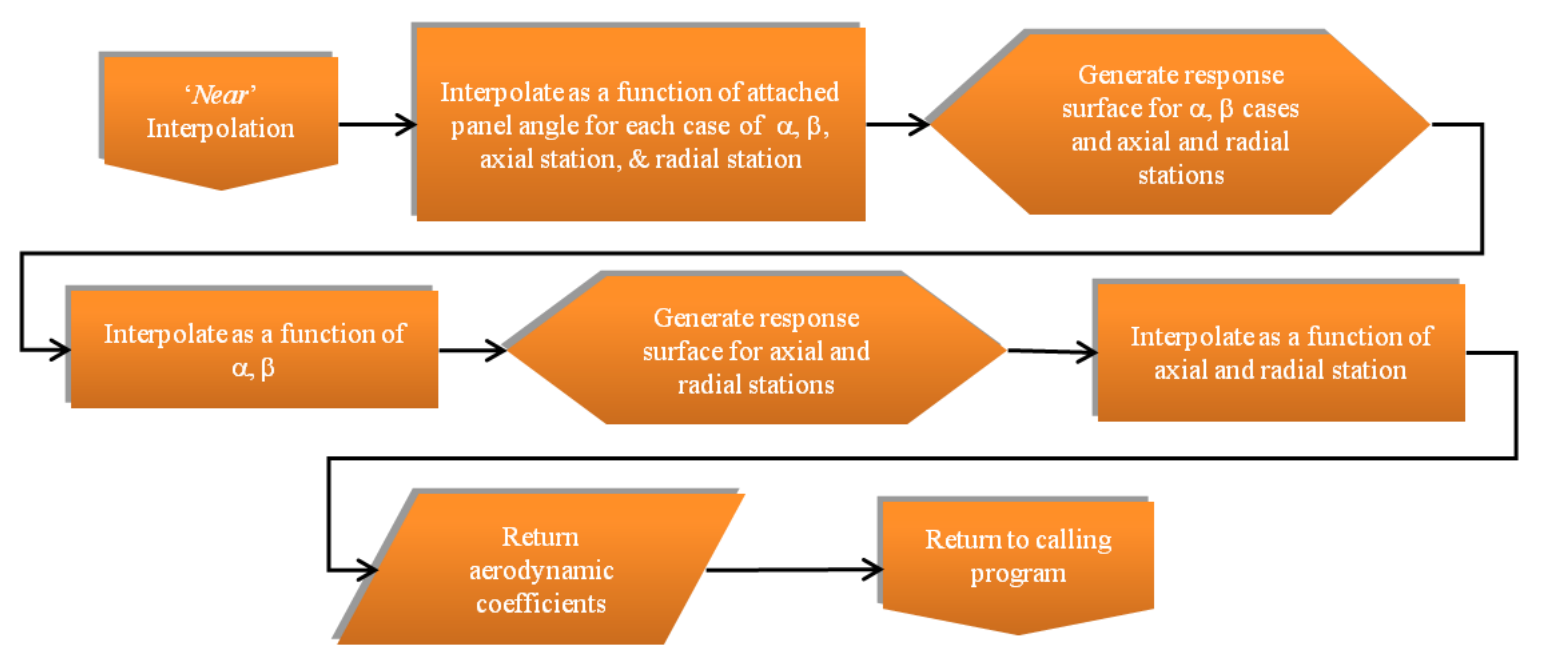

Figure 16: Flowchart of the near panel interpolation execution.

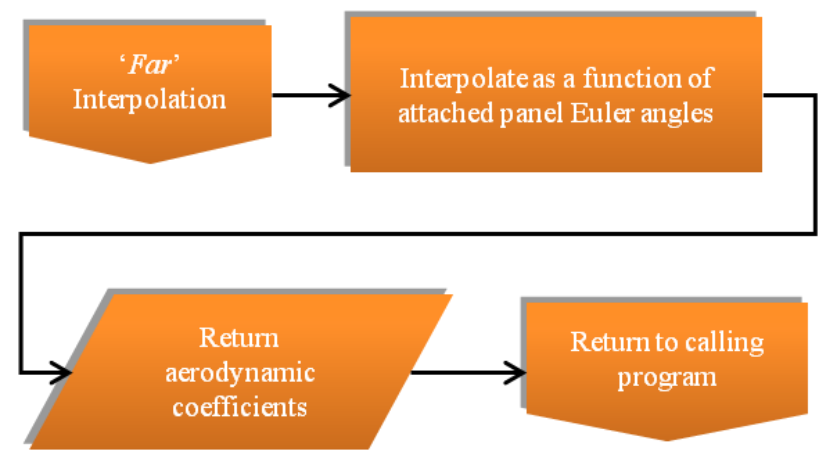

Figure 17: Flowchart of the far panel interpolation execution.

\section{Coupled SM Panel Data/6-DOF Simulations}

Testing of the interpolated aerodynamic coefficients was accomplished by coupling the data, MATLAB ${ }^{\circledR}$ scripts, and utilities with CRM's 6-DOF package and integrating in time (with proper constraints) to simulate the jettison event. These results were compared to CRM's QUICDyn simulation utility. QUICDyn (Quasi Unsteady Inviscid Dynamics) loosely couples CRM's 6-DOF solver directly with Cart3D to perform approximate moving body simulations. Simulations were performed for the four database design cases presented in Table 2 and an additional case with averaged freestream conditions, namely Mach 7, $\alpha=-18, \beta=0$. The last case tests the ability of the MATLAB script to interpolate the data for input parameters other than the database design parameters.

Figure 18 shows a comparison between the panel trajectories for the interpolated aerodynamic analysis tool and QUICDyn (shown as Cart3d in the plots). The aerodynamic database was designed for use with a dynamic pressure of $\mathrm{q}=0.5 \mathrm{psf}$ or lower. These simulations were run with the maximum dynamic pressure of $\mathrm{q}=0.5 \mathrm{psf}$. We note that the windward panel (2) is of primary interest in these plots since it always travels closest to the body and is the limiting factor in terms of panel clearance. The plot for Case 1 shows very good agreement between the interpolated result and QUICDyn for the side panel (3) and the windward panel (2). The leeward panel (1) exhibits a slight difference further downstream near the aft end of the vehicle. Case 2 displays more difference in the side panel (3) compared to Case 1 due to the change in side slip angle. However the panel of interest (2) still shows excellent agreement. For Case 3 all four of the panels travel in nearly identical radial distance trajectories. For this case, there is negligible difference between the database and QUICDyn. Case 4 again shows excellent agreement between the side panel (3) and the windward panel (2). The leeward panel (1) does exhibit some difference between the two methods. Case 5 is the averaged freestream condition simulation. This case shows very good 
agreement for the windward panel (2) and the side panel (3). However, the leeward panel (1) results diverge slightly near the front frustum.

We note that the differences in panel trajectories shown in Figure 14 are primarily due to the difference in the fidelity of the CFD solution for each method. The database method utilizes CFD mesh sizes between 3 and 6 million cells that are adapted to a composite functional of panel force coefficients. The QUICDyn approach uses non-adapted meshes with approximately 3 million cells. As a result, the QUICDyn approach would be expected to be less accurate. To illustrate this fact, Cases 1 and 2 were reran with a refined mesh for the QUICDyn approach. Figure 19 shows a comparison of QUICDyn versus the interpolated aerodynamic analysis tool for Case 1 and Case 2 at a dynamic pressure of $\mathrm{q}=1.0 \mathrm{psf}$. Note that a larger value of dynamic pressure was used to make aerodynamic effects more apparent. Two QUICDyn simulations are shown in the figure. The baseline QUICDyn solution is similar to the solutions presented in the previous section with computational grids of approximately 3 million cells. The refined QUICDyn solution uses 9 million cells with the majority of the clustering occurring in the vicinity of the cavity region. From the figure, we see that as we improve the QUICDyn grid, the solution approaches our interpolated results as expected. While the aerodynamic database was designed for use with a dynamic pressure of $\mathrm{q}=0.5 \mathrm{psf}$ or lower, the database is shown to produce excellent results at dynamic pressures above $\mathrm{q}=0.5 \mathrm{psf}$.

A single trajectory prediction obtained from the database required less than 5 minutes to execute on a single processor of a workstation. By comparison, a single trajectory coarse QUICDyn simulation required approximately 3 days on four processors for the same number of physical time steps. To obtain a single adaptive mesh QUICDyn trajectory prediction directly comparable to the database fidelity would require approximately six weeks on four processors. The efficiency of the database approach allows the researcher to examine the effects of decreasing the physical time step on the solution procedure. Furthermore researchers can rapidly investigate variant flight conditions and design changes such as panel weight, inertial properties, and mechanical spring force.

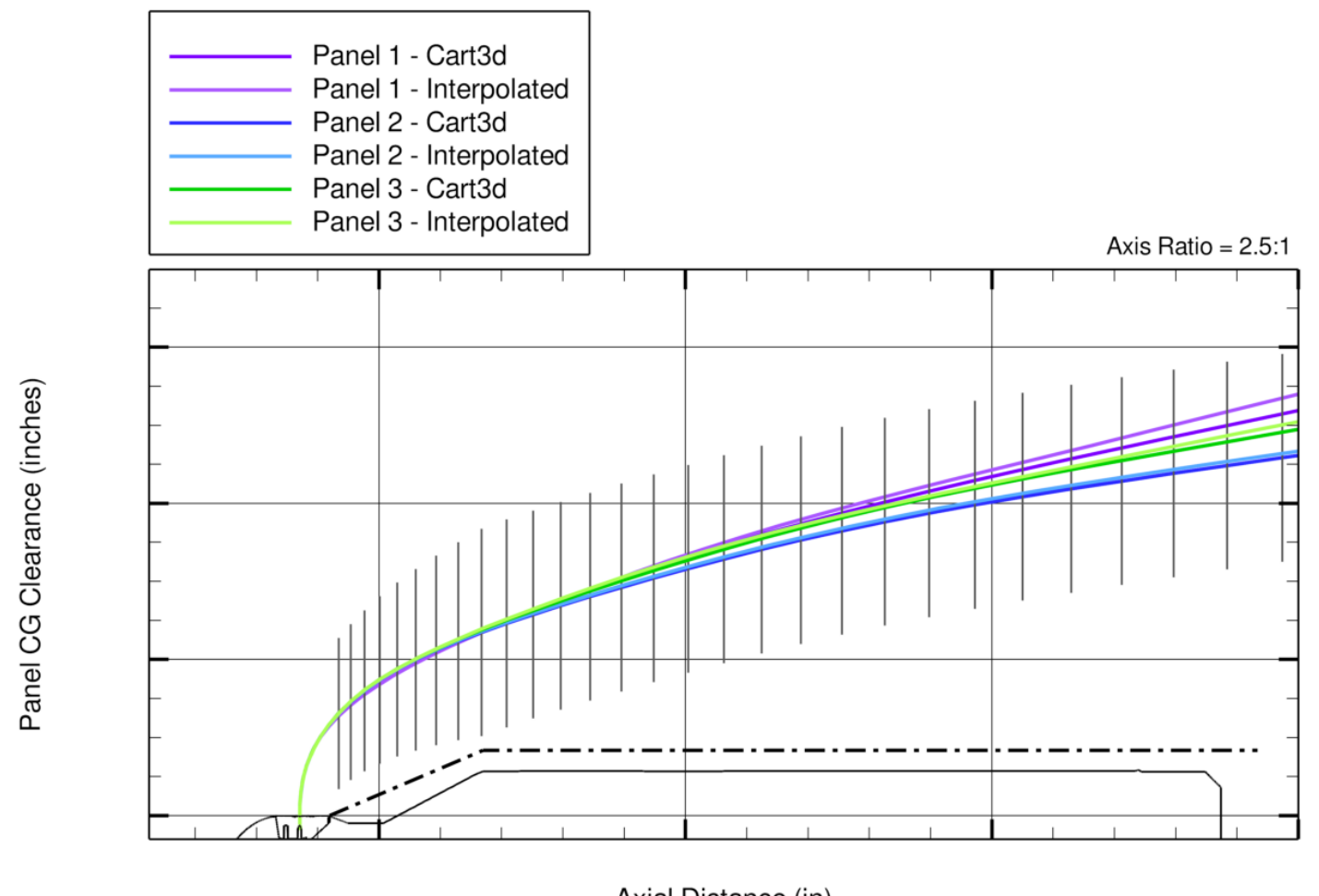

(a) Case 1: $\operatorname{Mach}=7, \alpha=-21, \beta=-5$ 


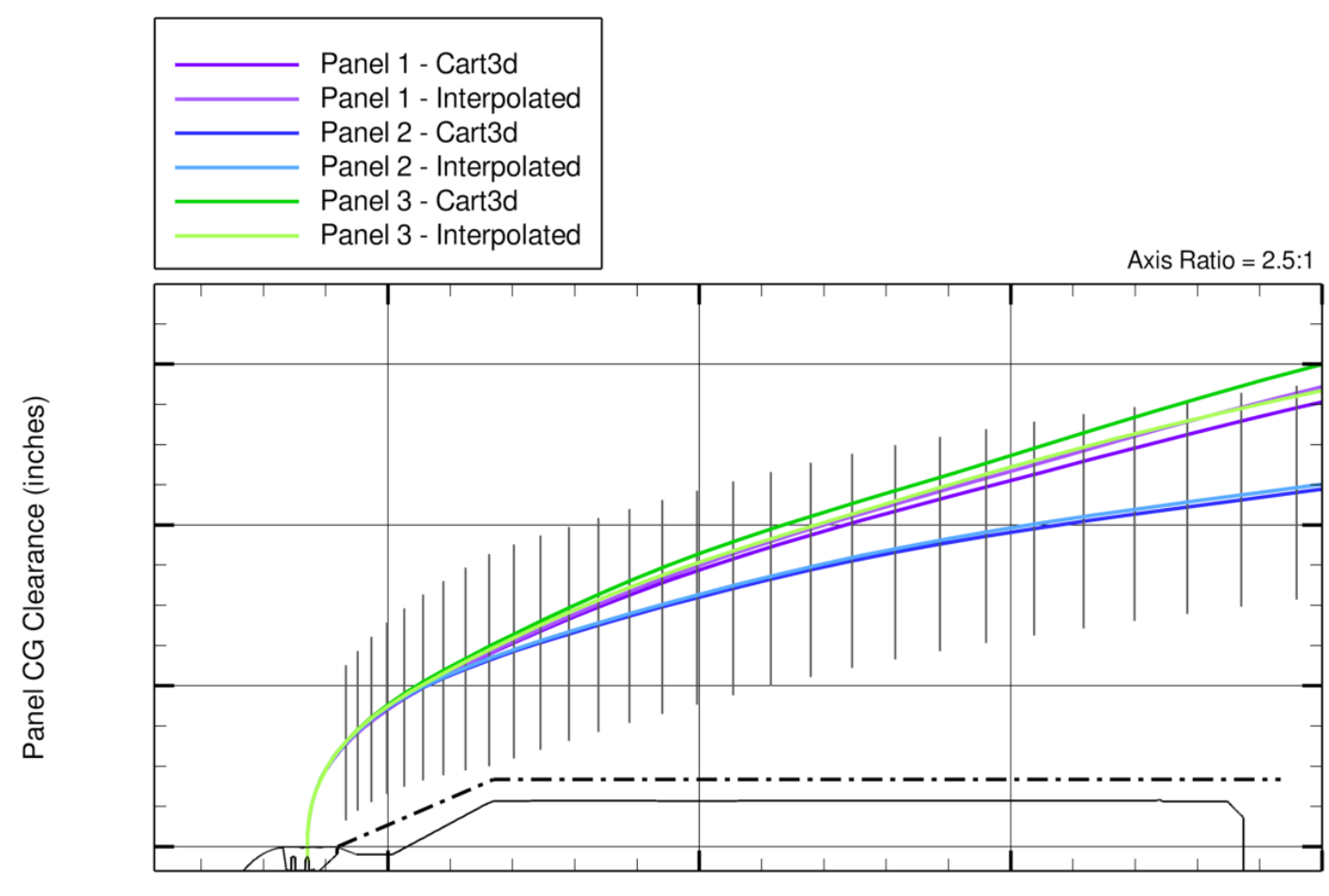

Axial Distance (in)

(b) Case 2: $\operatorname{Mach}=7, \alpha=-21, \beta=+5$

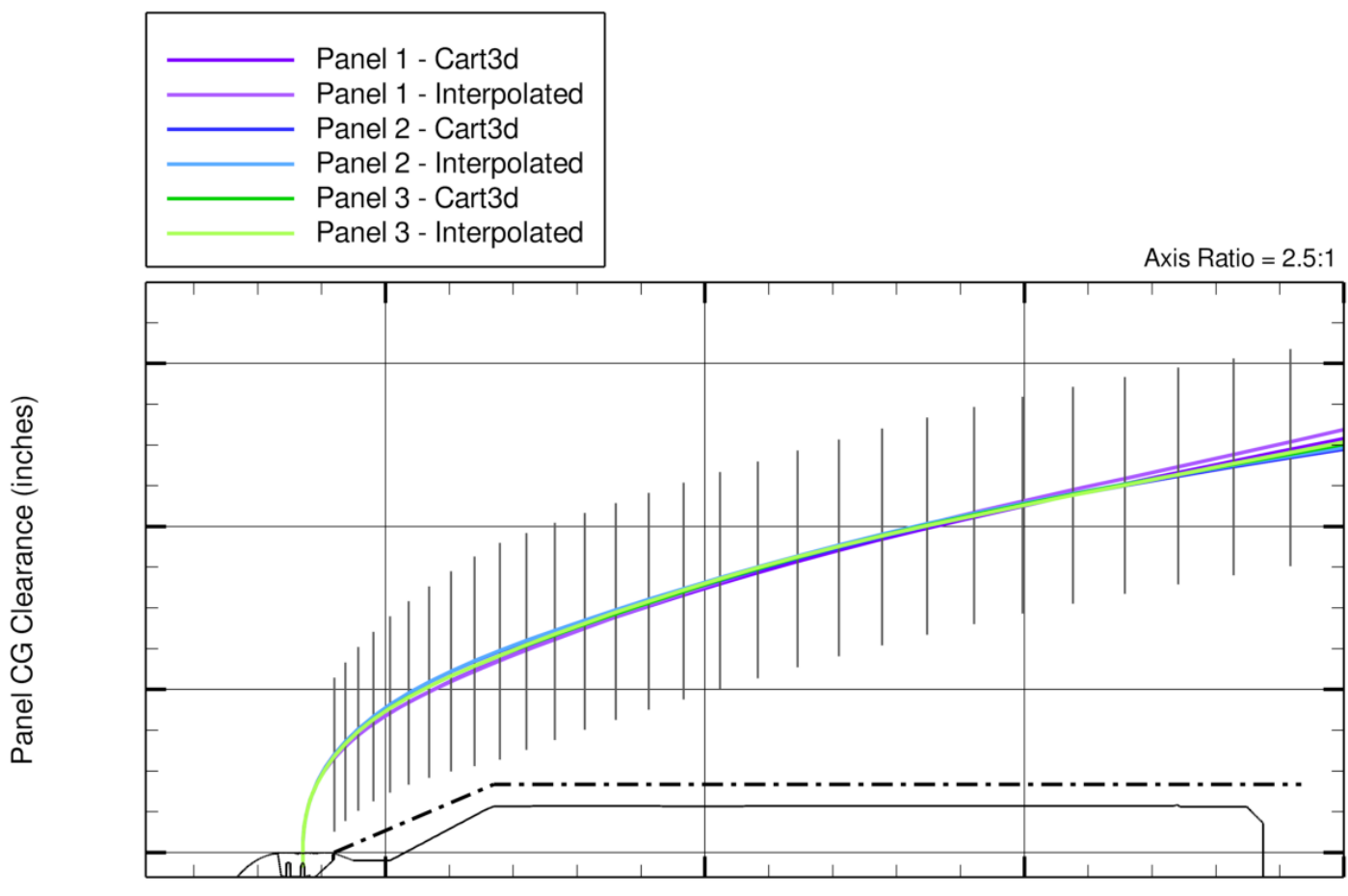

Axial Distance (in)

(c) Case 3: $\operatorname{Mach}=7, \alpha=-15, \beta=-5$

American Institute of Aeronautics and Astronautics 


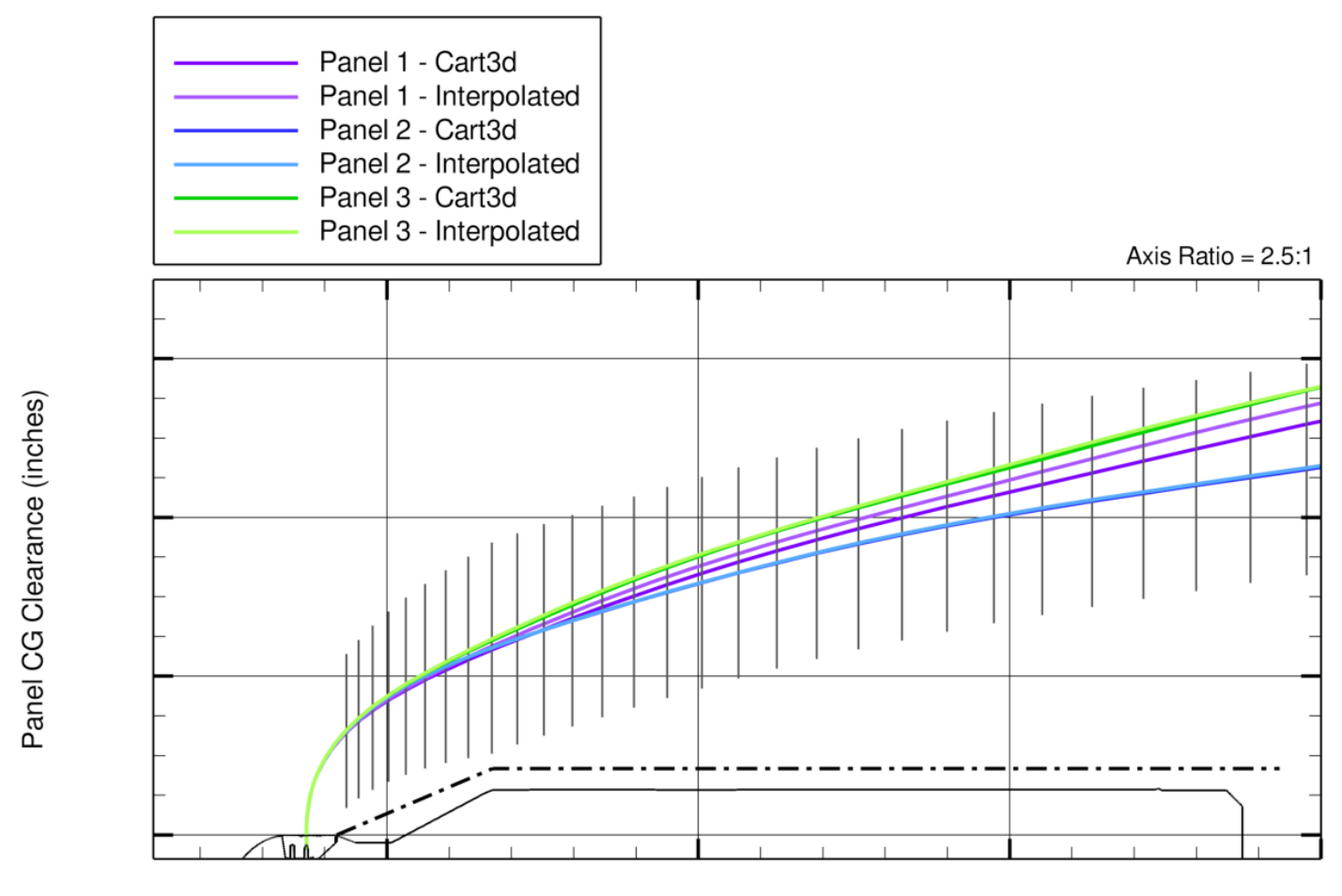

Axial Distance (in)

(d) Case 4: $\operatorname{Mach}=7, \alpha=-15, \beta=+5$

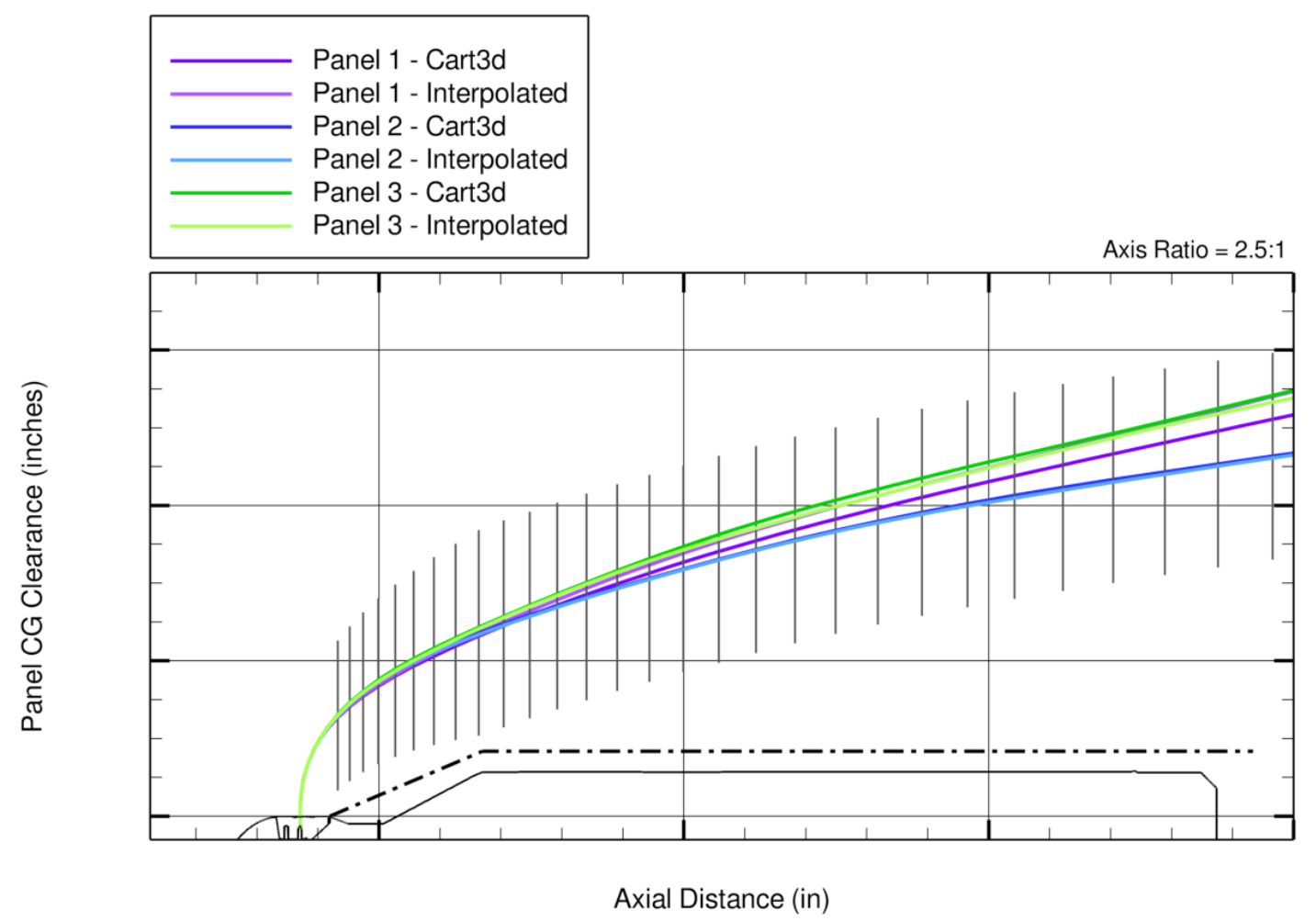

(e) Case 5: Mach $=7, \alpha=-18, \beta=0$ (averaged freestream conditions)

Figure 18 Comparison of the interpolated aerodynamic analysis tool with CRM's QUICDyn software package for the four database design cases and an averaged condition test case.

American Institute of Aeronautics and Astronautics 


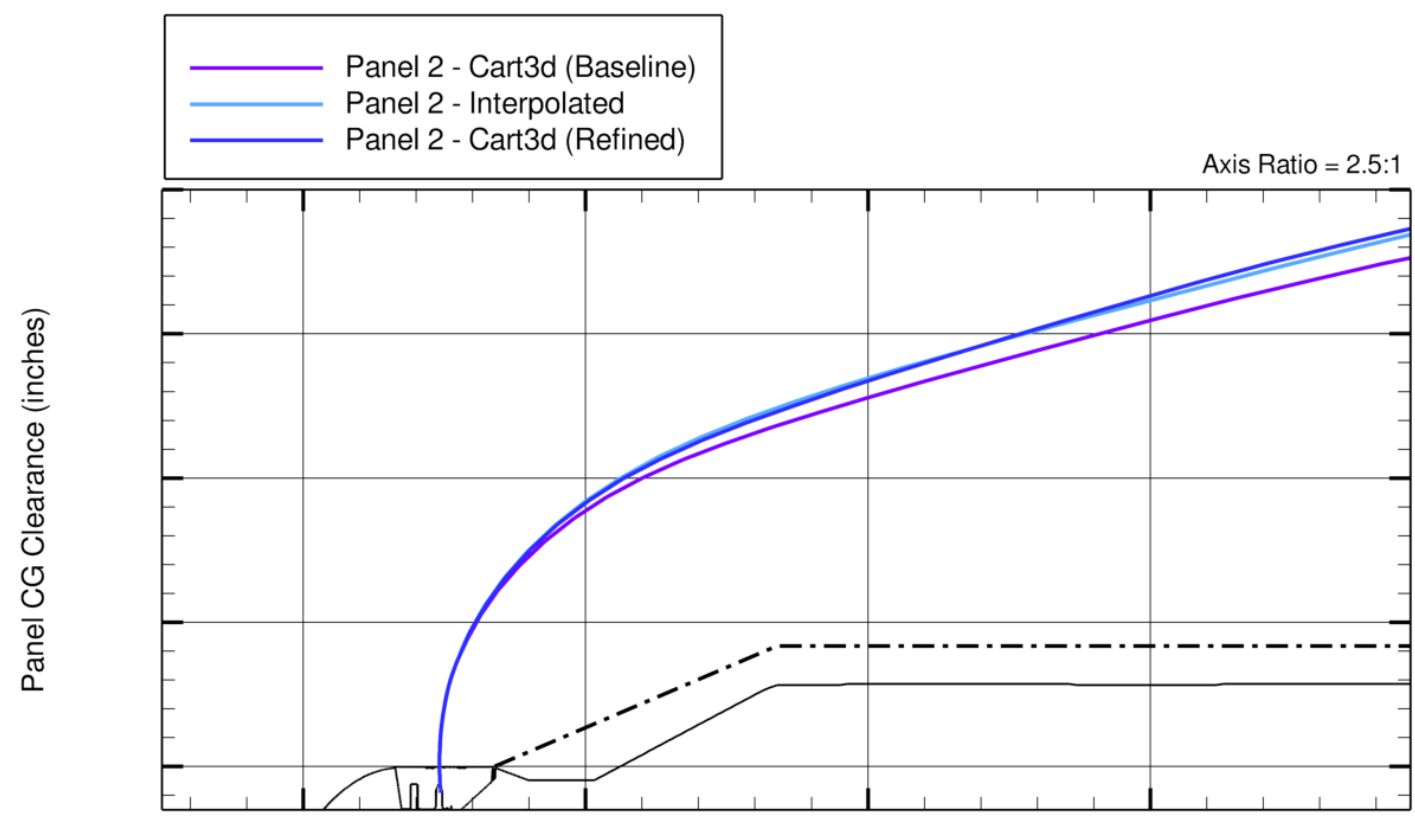

Axial Distance (in)

(a) Case 1: $\operatorname{Mach}=7, a=-21, b=-5$

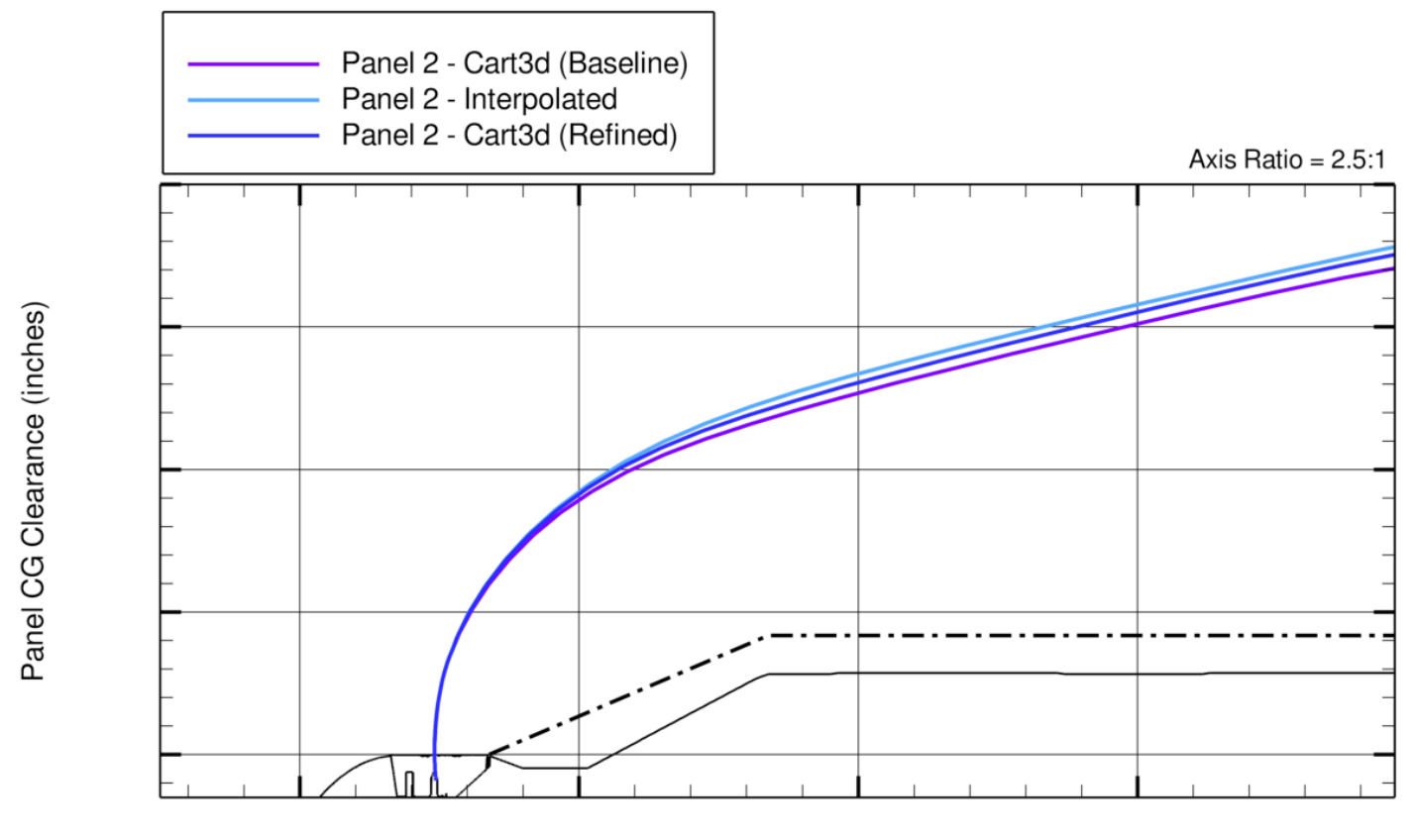

Axial Distance (in)

(b) Case 2: $\operatorname{Mach}=7, a=-21, b=+5$

Figure 19: Comparison of the interpolated aerodynamic analysis tool with CRM's QUICDyn software package at a dynamic pressure of $1.0 \mathrm{psf}$.

21

American Institute of Aeronautics and Astronautics 


\section{Conclusions}

A method for prediction of the SM panel jettison trajectories through utilization of an inviscid solver coupled with a 6-DOF model has been presented. Focus is on the windward panel which presents the greatest chance of recontact with the vehicle after separation. It is shown that forces and moments are captured with success using an inviscid solver when compared to higher fidelity viscous computations due to the physics of hypersonic flow exhibited on the windward panel. It is demonstrated that the aerodynamic effects of the SLS body cannot be ignored in such predictions at expected flight conditions. The pressurization of the cavity beneath the panel due to the aerodynamic environments provides an increased angular momentum imparted to the panel which provides additional clearance of the panel from the body. The initial force and moment predictions while the panel is on the hinge are most critical to obtaining the correct panel trajectories.

A database for SM panel jettison has been completed, with a total of 5144 CFD simulations (1448 hinged, 1512 near body, 2184 panel alone). The complete database due to mirroring of solutions contains 18,144 CFD simulations. Comparisons for windward panel trajectories show excellent agreement between quasi-unsteady analysis and the database. The subsequent database allows the user to perform a single trajectory prediction in less than 5 minutes on a single processor. A single loosely coupled adaptive mesh inviscid trajectory solution of the same fidelity could require as much as 6 weeks per simulation on four processors. The efficiency of the database approach allows the researcher to examine variant flight conditions and design changes such as panel weight, inertial properties, and mechanical spring force, etc., in a timely manner.

\section{References}

${ }^{1}$ Hall, L.H., Eppard, W.M., Applebaum, M.P., Mitchell, C.R., "Computational Simulation Techniques of Panel Fairing Jettison from a Launch Vehicle System”, JANNAF 959, 2008.

${ }^{2}$ Hall, L.H., Applebaum, M.P., Eppard, W.M.,"Debris Transport Modeling Techniques on Launch Vehicle Systems",49th AIAA Aerospace Sciences Meeting, Orlando, Fl, Jan 4-7, 2011.

${ }^{3}$ Murman, S.M., Aftosmis, M.J., Berger, M.J. "Simulations of 6-DOF Motion with a Cartesian Method", AIAA2003-1246, 41st AIAA Aerospace Sciences Meeting, Reno, NV, Jan 6-9, 2003.

${ }^{4}$ Hall, L.H., Parthasarathy, V., "Validation of an Automated Chimera/6-DOF Methodology for Multiple Moving Body Problems", AIAA 98-0753, 1998

${ }^{5}$ Aftosmis, M.J., Berger, M.J., and Adomavicius, G. "A Parallel Multilevel Method for Adaptively Refined Cartesian Grids with Embedded Boundaries”, AIAA-2000-0808, January 2000.

${ }^{6}$ Aftosmis, M.J., Berger, M.J., and Melton, J.E., "Robust and Efficient Cartesian Mesh Generation for Component-Based Geometry", AIAA Journal, Vol. 36, No. 6, pp. 952-960, 1998.

${ }^{7}$ Luke, E. A. and George, T., "Loci: A Rule-Based Framework for Parallel Multidisciplinary Simulation Synthesis", Journal of Functional Programming, Special Issue on Functional Approaches to High Performance Parallel Programming, Volume 15, Issue 03, 2005, pp. 477-502, Cambridge University Press.

${ }^{8}$ Luke, E. A., Tong, X.-L., Wu, J., and Cinnella, P., "CHEM 2: A Finite-Rate Viscous Chemistry Solver - The User Guide,” Tech. Rep. MSSU-COE-ERC-04-07, Mississippi State University, 2004. 